\title{
A computational model based on cortico-spinal fMRI for asymmetrically organized motor corticospinal networks in humans
}

\section{Eiji Takasawa}

National Center of Neurology and Psychiatry

Mitsunari Abe ( $\square$ mitzabe@gmail.com )

National Center of Neurology and Psychiatry https://orcid.org/0000-0002-3913-2292

\section{Kenji Takagishi}

Gunma University Graduate School of Medicine,

\section{Hirotaka Chikuda}

Gunma University Graduate School of Medicine,

\section{Takashi Hanakawa}

Kyoto University Graduate School of Medicine,

\section{Article}

Keywords: The direct and indirect corticospinal motor pathway, laterization, hand preference, corticospinal functional magnetic resonance imaging

Posted Date: March 16th, 2021

DOl: https://doi.org/10.21203/rs.3.rs-308675/v1

License: (c) (1) This work is licensed under a Creative Commons Attribution 4.0 International License. Read Full License

Version of Record: A version of this preprint was published at Communications Biology on July 5th, 2022. See the published version at https://doi.org/10.1038/s42003-022-03615-2. 
Title: A computational model based on cortico-spinal fMRI for asymmetrically organized motor corticospinal networks in humans

Authors: Eiji Takasawa ${ }^{1,2} \uparrow$, Mitsunari Abe ${ }^{1 * \dagger} \uparrow$, Kenji Takagishi ${ }^{2}$, Hirotaka Chikuda ${ }^{2}$, Takashi Hanakawa ${ }^{3,1 *}$

\section{Affiliations:}

${ }^{1}$ Department of Advanced Neuroimaging, Integrative Brain Imaging Center, National Center of Neurology and Psychiatry, Tokyo, Japan

${ }^{2}$ Department of Orthopaedic Surgery, Gunma University Graduate School of Medicine, Maebashi, Gunma, Japan.

${ }^{3}$ Department of Integrated Neuroanatomy \& Neuroimaging, Kyoto University Graduate School of Medicine, Kyoto, Japan

*Correspondence to: Mitsunari Abe (mitsunari.abe.a8@ncnp.go.jp) or Takashi Hanakawa (hanakawa.takashi.2s@kyoto-u.ac.jp)

$\dagger$ indicates equal contributions to this work.

Key words: The direct and indirect corticospinal motor pathway, laterization, hand preference, corticospinal functional magnetic resonance imaging

Classification: Biological Sciences 


\begin{abstract}
:
Evolution of the direct connection from primary motor cortex to motoneurons in the spinal cord parallels acquisition of hand dexterity and lateralization of hand preference. Recent studies indicated that the phylogenetically older pathway consisting of multi-synaptic connections from primary motor cortex to spinal motoneurons also participate in controlling dexterous hand movement. However, it remains unknown how the two corticospinal pathways work in concert to control unilateral hand movement with lateralized preference. Using corticospinal functional magnetic resonance imaging, we discovered the asymmetric organization of the two corticospinal networks that modelled monosynaptic or polysynaptic control from primary motor cortices over spinal motoneurons. Moreover, the degree of the involvement of the two corticospinal networks paralleled the lateralization of hand preference. The present results pointed to the functionally lateralized motor nervous system that underlies the behavioural asymmetry of handedness, a uniquely human trait which could have phylogenetically differentiated humans from other primates.
\end{abstract}




\section{Introduction}

Hand dexterity is a remarkable ability characterizing higher primates, including humans, who consistently use a preferred hand to perform manual dexterity tasks in daily activities ${ }^{1}$. For unknown reasons, the acquisition of hand dexterity parallels lateralization of hand preference. Hand movement is controlled mainly by primary motor cortex (M1) ${ }^{1}$. M1 contralateral to the moving hand sends the direct corticomotoneuronal connection to motoneurons in the spinal cord ${ }^{1}$. M1s in bilateral hemispheres connect to the multi-synaptic relay circuits in the brainstem and the spinal cord that finally terminate on motoneurons ${ }^{1}$. The corticomotoneuronal connection appears in higher primates, and plays a major role in control of hand dexterous movement. Although the relay connections in the brainstem and the spinal cord had long been considered necessary for gross forelimb movements in lower mammals, recent evidence suggests that they also participate in controlling dexterous hand movement in higher primates ${ }^{1}$. This evidence pointed to the phylogenetically new and old models of the motor corticospinal networks that exert monosynaptic or polysynaptic control from M1s over spinal motoneurons ${ }^{2,3}$. Recent studies implicated the inherently asymmetric organization of the corticospinal motor pathways underlying unilateral RHM and $\mathrm{LHM}^{4,5}$. It has been proposed that this network asymmetry may be associated with behavioural laterality, indicating right-handedness in daily motor activities ${ }^{4,5}$. However, it remains unclear how the two corticospinal networks - that modelled monosynaptic or polysynaptic influences of M1s onto spinal motoneurons - work in concert to control dexterous hand movement. Moreover, it is unknown whether the organization of the two corticospinal networks relates to hand preference. Here, we applied corticospinal functional magnetic resonance imaging (fMRI) to right-handed humans to estimate recruitment of the two corticospinal networks during RHM or LHM. To estimate network connectivity, we computed the influences from M1s onto the spinal cord at the segments where motoneurons innervating hand muscles reside. We operationally called the 'direct' and 'non-direct' corticospinal networks that modelled monosynaptic or polysynaptic control from M1s over motoneurons in the spinal cord. We predicted recruitment of the direct corticospinal network during both of RHM and LHM while 
that of the non-direct corticospinal network during LHM in right-handed participants ${ }^{6-9}$. Moreover, we investigated if individual's differences in involvement of the direct and non-direct corticospinal networks paralleled the intrasubject variability of the degree of right-handedness. A behavioural laterality of handedness was assessed by the questionnaire of Edinburgh handedness inventory (EHI) ${ }^{10}$. We hypothesized the greater involvement of the direct and the non-direct corticospinal pathway in preferred and nonpreferred-hand movement that was correlated with the higher EHI scores indicating the greater degree of right-handedness.

\section{Results}

\section{Asymmetry in activation patterns of the spinal cord and M1 during RHM and LHM}

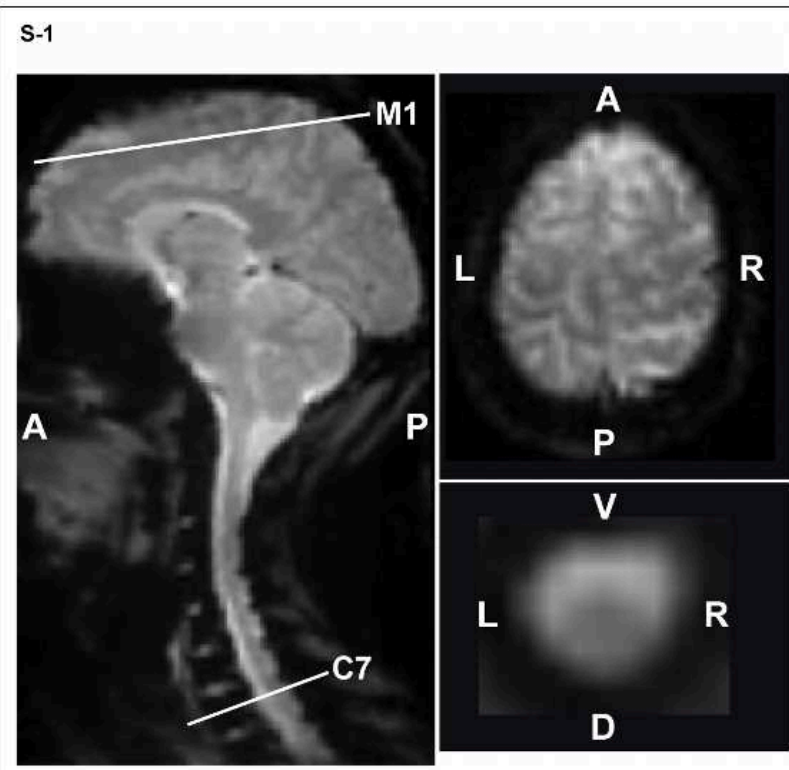

S-1. Echo planar imaging acquired with multiple slices along a sagittal plane. This protocol allowed the scanning of regions of interest in one single volume including M1 in bilateral hemispheres and the cervical spinal cord. The left panel shows the sagittal planes along the longitudinal body axis (i.e. rostro-caudal) covering the brain hemisphere and the spinal cord. $\mathrm{A}=$ anterior side. $\mathrm{P}=$ posterior side. Right panels show axial images of bilateral M1s (see the while line at the level of hand area in M1, left panel) and the spinal cord (see while line at the segmental level of $\mathrm{C} 7$, left panel) that were reconstructed from the raw data consisting of the sagittal slices. $L=$ left side. $R=$ right side. Upper and lower side of the axial images correspond to the anterior and posterior side of the brain or the ventral and dorsal side of the spinal cord. $\mathrm{V}=$ ventral side. $\mathrm{D}=$ dorsal side. For display purposes, the axial view of the reconstructed images has been magnified by 3 .
Thirteen young healthy adults performed a finger opposition task with RHM or LHM during the simultaneous measurement of blood oxygenation level-dependent (BOLD) signals from bilateral M1 and the spinal cord (see supplementary data S-1). First, we examined activity in M1s and the spinal cord at the level of C7-Th1 segments during RHM or LHM. Activity data was used to estimate network connectivity between $\mathrm{M} 1$ and the spinal cord in the following analyses. BOLD activity in the right or left half of the spinal cord (SHc) replicated the findings of previous spinal fMRI studies ${ }^{11-13}$. SHc activity was computed as

averaged among BOLD signals in right or left SHc at the level of the $\mathrm{C} 5$ or C7-Th1 segments. The 


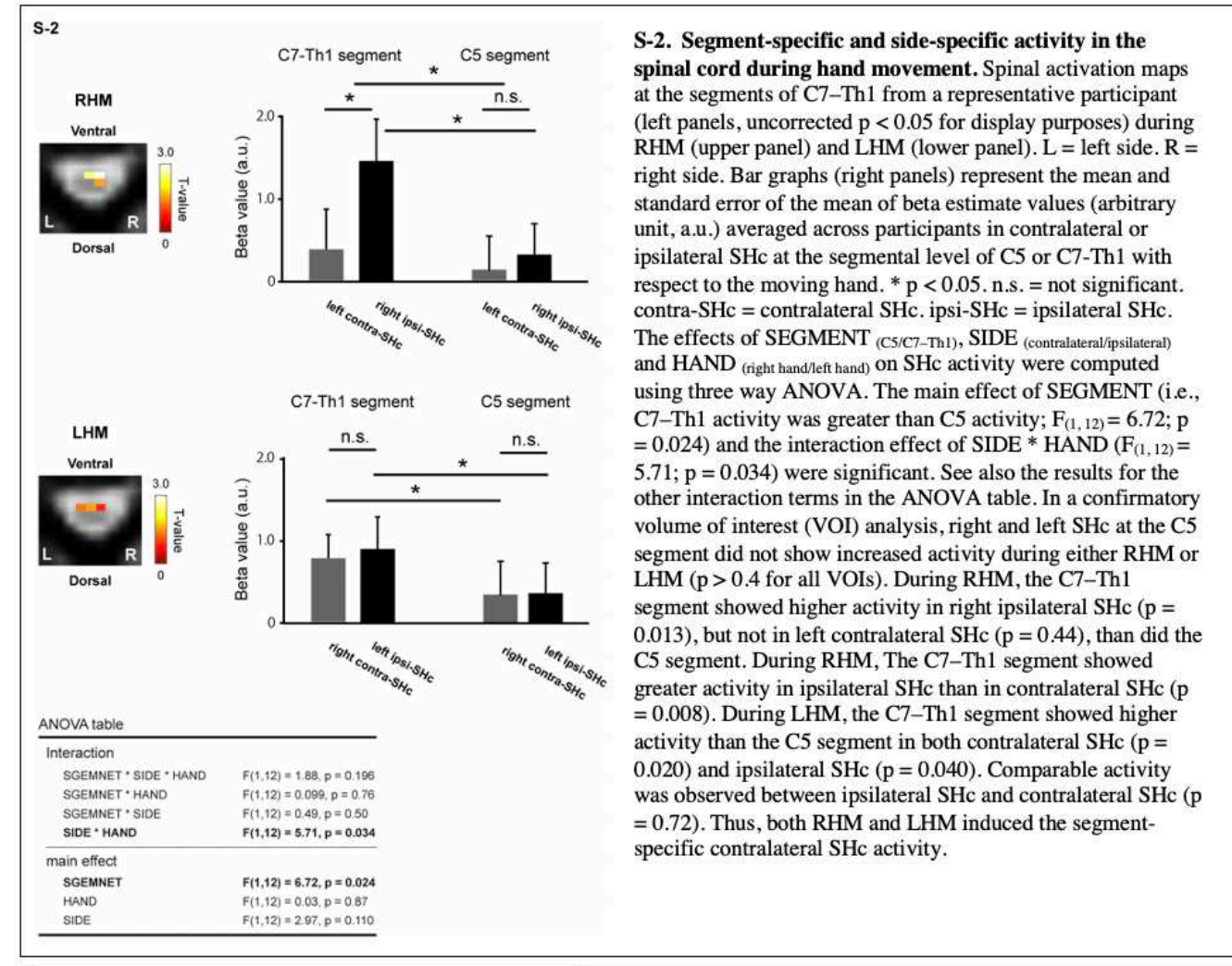

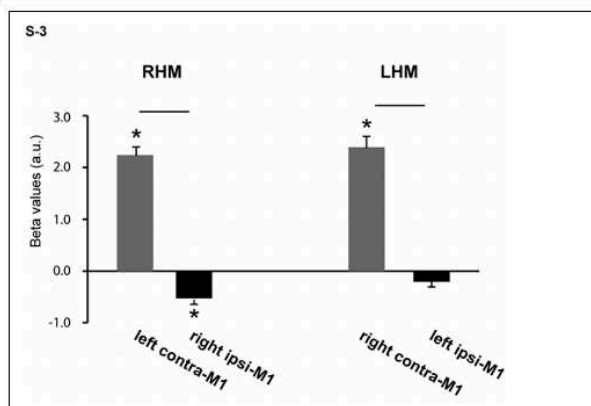

S-3. Activity in primary motor cortex (M1) contralateral and ipsilateral to the moving hand. Voxel-wise, whole-brain analysis was used to investigate activity in $\mathrm{M} 1$ in both hemispheres. RHM and LHM activated contralateral M1 as compared to rest. For left M1 during RHM, peak MNI coordinates $(x, y, z)=-34,-20,58, T=7.94$, family-wise error (FWE) corrected $\mathrm{p}=0.03$. For right $\mathrm{M} 1$ during LHM, peak MNI coordinates $(x, y, z)=38,-16,52, T=7.85$, FWE corrected $p=$ 0.03 . To estimate ipsilateral M1 activity, analysis was performed in the spherical VOIs with $8 \mathrm{~mm}$ radius centred at the peak coordinate of left M1 or right M1. RHM suppressed activity in right ipsilateral $\mathrm{M} 1$ relative to rest $(\mathrm{T}=5.35, \mathrm{FWE}$ corrected $\mathrm{p}=$ 0.04 ), while LHM did not induce suppression of left ipsilateral M1 activity (uncorrected $p>0.1$ ). VOI analysis was used to examine different activities between bilateral M1 in the side contralateral or ipsilateral to the moving hand. The beta estimate values were computed within the $8 \mathrm{~mm}$ sphere centred at the peak coordinate of left or right M1. Bar graphs represent the mean and standard error of the mean of beta estimate values (arbitrary unit, a.u.) averaged across participants in contralateral or ipsilateral M1 with respect to the moving hand. $* \mathrm{p}<0.05$. contra-M1 $=$ contralateral M1. ipsi-SHc $=$ ipsilateral M1. Activity was higher in contralateral M1 than in ipsilateral M1 during either RHM or LHM (paired t-test of left M1 vs. right M1 during RHM, $\mathrm{p}=$ 0.0002 ; paired t-test of left $\mathrm{M} 1 \mathrm{vs}$. right $\mathrm{M} 1$ during LHM, $\mathrm{p}=$ 0.0002 ). Reduction of activity in ipsilateral M1 during RHM was greater than during LHM (paired t-test of left $\mathrm{M} 1 \mathrm{vs}$. right $\mathrm{M} 1, \mathrm{t}_{12}$ $=-2.54, \mathrm{p}=0.026$ ). regions of interest included both of grey and white matter (see Method). Motoneurons at C7-Th1 segments innervate to hand muscles more dominantly than those at the C5 segment. We tested higher activity in ipsilateral SHc at the level of C7-Th1 segments than at the level of C5 segment. We computed the effects of SEGMENT (C5 versus C7-Th1), SIDE (contralateral SHc versus ipsilateral SHc) and HAND (RHM versus LHM) on SHc activity using a three-way analysis of variance (ANOVA) (S-2). Multiple comparisons were performed with use of Bonferroni-Holm correction. The main effect of SEGMENT (i.e., C7-Th1 activity was greater than $\mathrm{C} 5$ activity; $\mathrm{F}_{(1,12)}=$ $6.72 ; p=0.024)$ and the interaction effect of SIDE * HAND $\left(F_{(1,12)}=5.71 ; p=0.034\right)$ were significant. We found such

spinal segment-specific activity similarly for RHM and LHM. The SIDE-by-HAND interaction was 
significant only during RHM. In a confirmatory volume of interest (VOI) analysis, right and left SHc at the C5 segment did not show increased activity during either RHM or LHM ( $p>0.4$ for all VOIs, see right part of the upper and lower panels, S-2). During RHM, the C7-Th1 segments showed higher activity in right ipsilateral SHc $(p=0.013)$, but not in left contralateral SHc $(p=0.44)$, compared to the C5 segment. Moreover, the C7-Th1 segment showed greater activity in ipsilateral SHc than in contralateral SHc $(p=0.008)$. During LHM, the C7-Th1 segments showed higher activity than the C5 segment in both contralateral $(p=0.020)$ and ipsilateral SHc $(p=0.040)$. Comparable activity was observed between ipsilateral and contralateral SHc $(\mathrm{p}=0.72)$. These results showed a segment-specific activity in ipsilateral SHc during RHM or LHM. Previous reports also replicated activation of not only the ipsilateral SHc but also the contralateral SHc during LHM, possibly recruitment of interneurons that contact on motoneurons in ipsilateral SHc ${ }^{14,15}$. BOLD activity in M1 (S-3) confirmed from the findings of previous studies ${ }^{16,17}$. M1 activity was computed as averaged among BOLD signals in activated voxels inside Brodmann area 4 (see Method). Both RHM and LHM mainly activated contralateral M1 (family-wise error [FWE] corrected $\mathrm{p}<0.05$ ). Moreover, RHM deactivated ipsilateral M1 relative to the resting baseline (corrected $\mathrm{p}=0.001$ by small volume correction analysis), whereas LHM did not $(\mathrm{p}>0.1)$. Deactivation of ipsilateral M1 was evident during RHM than during LHM (paired t test, left M1 vs. right M1, $\mathrm{t}_{12}=-2.54$, $\mathrm{p}=0.026)$. These results replicated the previous findings of independently measured M1 and SHc activities during unilateral hand movement, endorsing the present simultaneous corticospinal fMRI methodology ${ }^{11-13,16-18}$. 
Asymmetric recruitment of the network during LHM consisting of the spinal cord and M1, both of which are ipsilateral to the moving hand

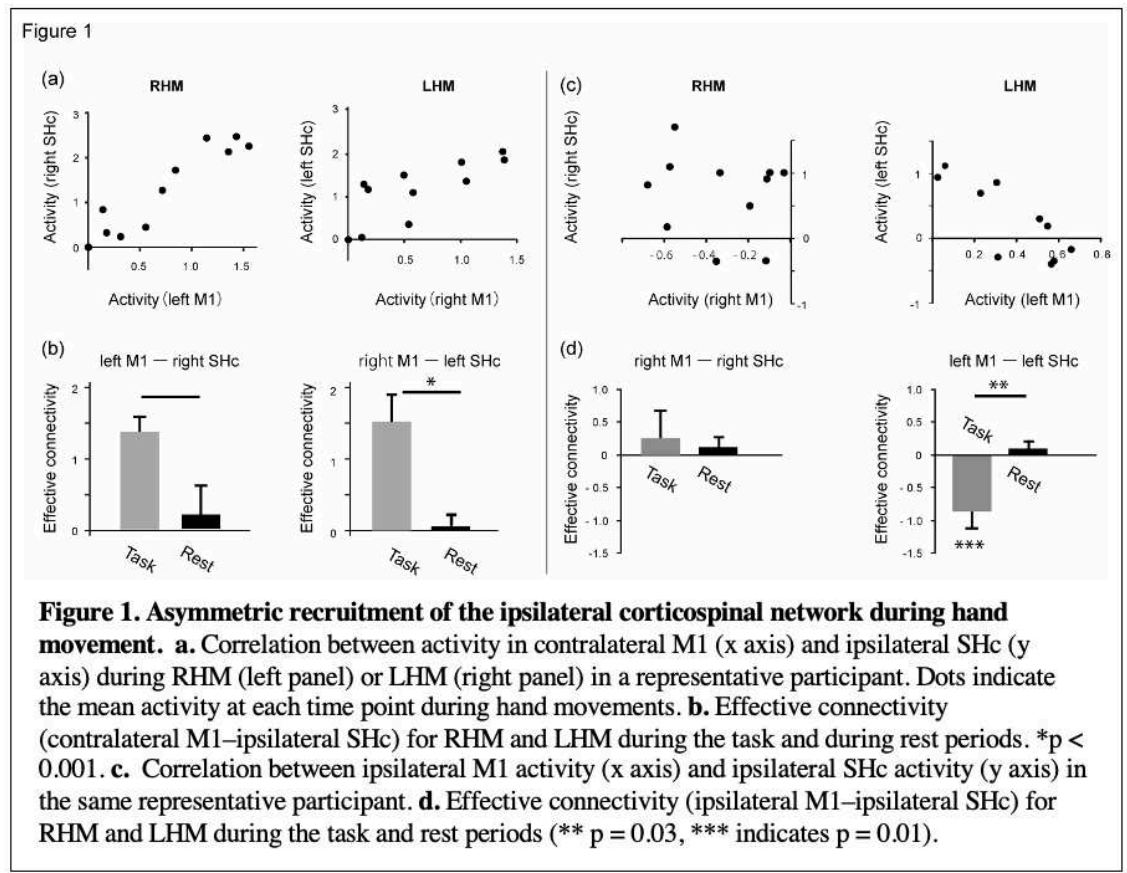

Previous reports indicated that both RHM and LHM involved the network between contralateral M1 and ipsilateral SHc (contralateral M1 - SHc network) while LHM (but not RHM) recruited the network between ipsilateral M1 and ipsilateral SHc (ipsilateral M1- SHc network) ${ }^{7,8}$. We analyzed the taskinduced modulation of effective connectivity between M1 and SHc, which provides an estimation of an input-output function. A simple linear regression analysis was performed on each individual's data,
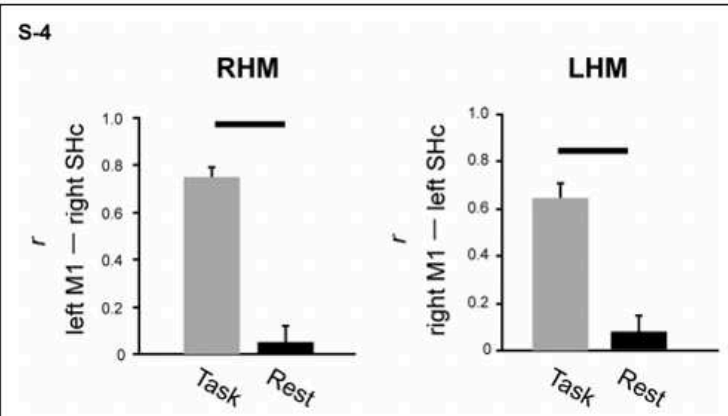

S-4. Correlation coefficient in the contralateral M1-SHc network during hand movement relative to rest.

Bar graphs represent the mean and standard error of the mean of the correlation coefficient $(r)$ between contralateral M1 activity and ipsilateral SHc activity during hand movement or rest (RHM, $r=0.75 \pm 0.04$; rest, $r=0.09 \pm 0.11$. LHM, $r=0.64 \pm$ 0.06 ; rest, $r=0.014 \pm 0.09$ ). Correlation coefficient $(r)$ was higher during hand movement relative to rest, regardless of the moving hand (RHM vs. rest, $\mathrm{p}=0.033$. LHM vs. rest, $\mathrm{p}=$ 0.043).

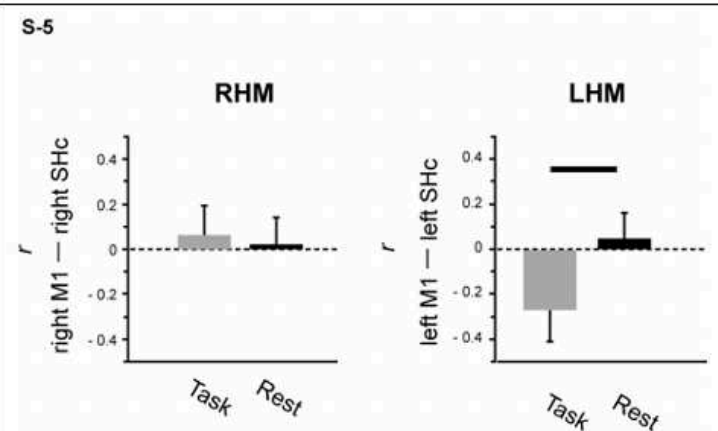

S-5. Correlation coefficient in the ipsilateral M1-SHc network during hand movement relative to rest. Bar graphs represent the mean and standard error of the mean of the correlation coefficient $(r)$ between ipsilateral M1 activity and ipsilateral SHc activity during hand movement or rest (RHM, movement, $r=0.065 \pm 0.13$; rest, $r=0.049 \pm 0.11$. LHM, movement, $r=-0.29 \pm 0.09$; rest, $r=0.06 \pm 0.10$ ). LHM increased the correlation coefficient $(r)$ in an inverse manner during LHM (movement vs. rest, $\mathrm{p}=0.010$ ), whereas RHM did not affect the coefficient (movement vs. rest, $p=0.78$ ). 
assigning M1 activity (S-3) as the explanatory variable and SHc activity at the level of C7-Th1 segments (S-2) as the independent variable (Fig. 1a). The regression slope was taken as a measure of effective connectivity ${ }^{19}$. Changes in effective connectivity were compared between RHM or LHM and at rest using a Wilcoxon signed-rank test. Functional connectivity was also computed to confirm the statistical correlation of activity (i.e., correlation coefficient) between M1 and SHc during RHM or LHM relative to rest (S-4 to S-5 and S-7). The effective connectivity of the contralateral M1-SHc network was positive during both RHM and LHM (Fig. 1b and S-4). These results support that both RHM and LHM involve the contralateral M1-SHc network that is subserved by excitatory projections ${ }^{1}$. The effective connectivity of the ipsilateral M1-SHc network did not change between RHM and rest (Fig. 1c, d and S-5). In contrast, the effective connectivity of the ipsilateral M1-SHc network was negative, demonstrating an inverse relationship between ipsilateral M1 activity and ipsilateral SHc activity during LHM (Fig. 1c and d). These results support the involvement of the ipsilateral M1-SHc network during LHM only ${ }^{7,8}$. This network exerts overall inhibitory influences onto motoneurons in humans ${ }^{20}$, which is probably underpinned by the combination of excitatory and inhibitory effects mediated by the reticulospinal, propriospinal and segmental interneuronal tracts ${ }^{21}$. Further analyses indicated that a higher contralateral M1 activity was coupled with a greater negative value of the effective connectivity of the ipsilateral M1\begin{tabular}{|l}
\hline S-6 \\
S-6. Contralateral M1 activity and effective connectivity of the \\
ipsilateral network between M1 and SHc. Relationship between \\
contralateral M1 activity (x axis) and effective connectivity of the \\
ipsilateral M1-ipsilateral SHc network (y axis), in which each dot \\
indicates a single participant's data. . The higher the right contralateral \\
M1 activity, the greater the negative value of the effective connectivity \\
of the ipsilateral M1-ipsilateral SHc network during LHM (right panel, \\
correlation coefficient, $r=-0.58, \mathrm{p}=0.03 ;$ simple linear regression \\
analysis, regression slope $=-0.58 ; 95 \%$ confidence intervals -0.57 to \\
0.13). b. Such a relationship was not evident between contralateral M1 \\
activity and effective connectivity of the ipsilateral M1-ipsilateral SHc \\
network during RHM (left panel, $\mathrm{p}=0.39$ ).
\end{tabular} SHc network during LHM $(r=-0.58, \mathrm{p}=0.03$; S6). This relationship was not clear, however, during RHM $(p=0.39)$. The dependency of contralateral 
S-7. Functional connectivity and effective connectivity between bilateral M1s. Simple correlation analysis was performed between bilateral M1 activity during hand movement or rest. The mean and standard error of the mean of the correlation coefficient $(r)$ are as follows: RHM, movement, $r=-0.07 \pm 0.17$; rest, $r=0.027 \pm 0.11$. LHM, movement, $r=-0.095 \pm 0.12$; rest, $r=0.013 \pm 0.08$. Task induced modulation of interhemispheric functional connectivity was not found during either hand movement (Wilcoxon signed-rank test, RHM, $p=0.55 ;$ LHM, $\mathrm{p}=0.57$ ).

Simple linear regression analysis was computed between bilateral M1 activity during hand movement or rest. The mean and standard error of the mean of the regression slope are as follows: RHM, slope $=-0.13 \pm 0.11$; rest, slope $=0.03 \pm$ 0.08 . LHM, slope $=-0.04 \pm 0.26$; rest, slope $=0.03 \pm 0.14$ ). Neither RHM nor LHM modulated effective connectivity between bilateral M1 (Wilcoxon signedrank test, RHM, $\mathrm{p}=0.38 ; \mathrm{LHM}, \mathrm{p}=0.50$ ).
M1 activity conforms to the idea that ipsilateral M1 - SHc network

receives efferent signals from

bilateral M1s during $\mathrm{LHM}^{22,23}$. It is

also possible that interhemispheric

connectivity between bilateral M1

could influence motor cortical-

spinal connectivity differently between RHM and LHM. Previously, it has been proposed that

interhemispheric influences between M1 might modulate the recruitment of the ipsilateral M1-SHc

network $^{24,25}$. We tested this possibility, but did not find any task-induced modulation of interhemispheric effective connectivity during either RHM or LHM ( $p>0.3$ for both hands; S-7). This finding is consistent with recent reports, whereby, although gross hand movements induce an increased effective connectivity between M1 hemispheres ${ }^{26,27}$, fine hand movements do not ${ }^{28}$. Therefore, it may be surmised that interhemispheric effective connectivity between M1 hemispheres was less likely to be involved in the modulation of M1-SHc networks in the finger tapping task tested here. 


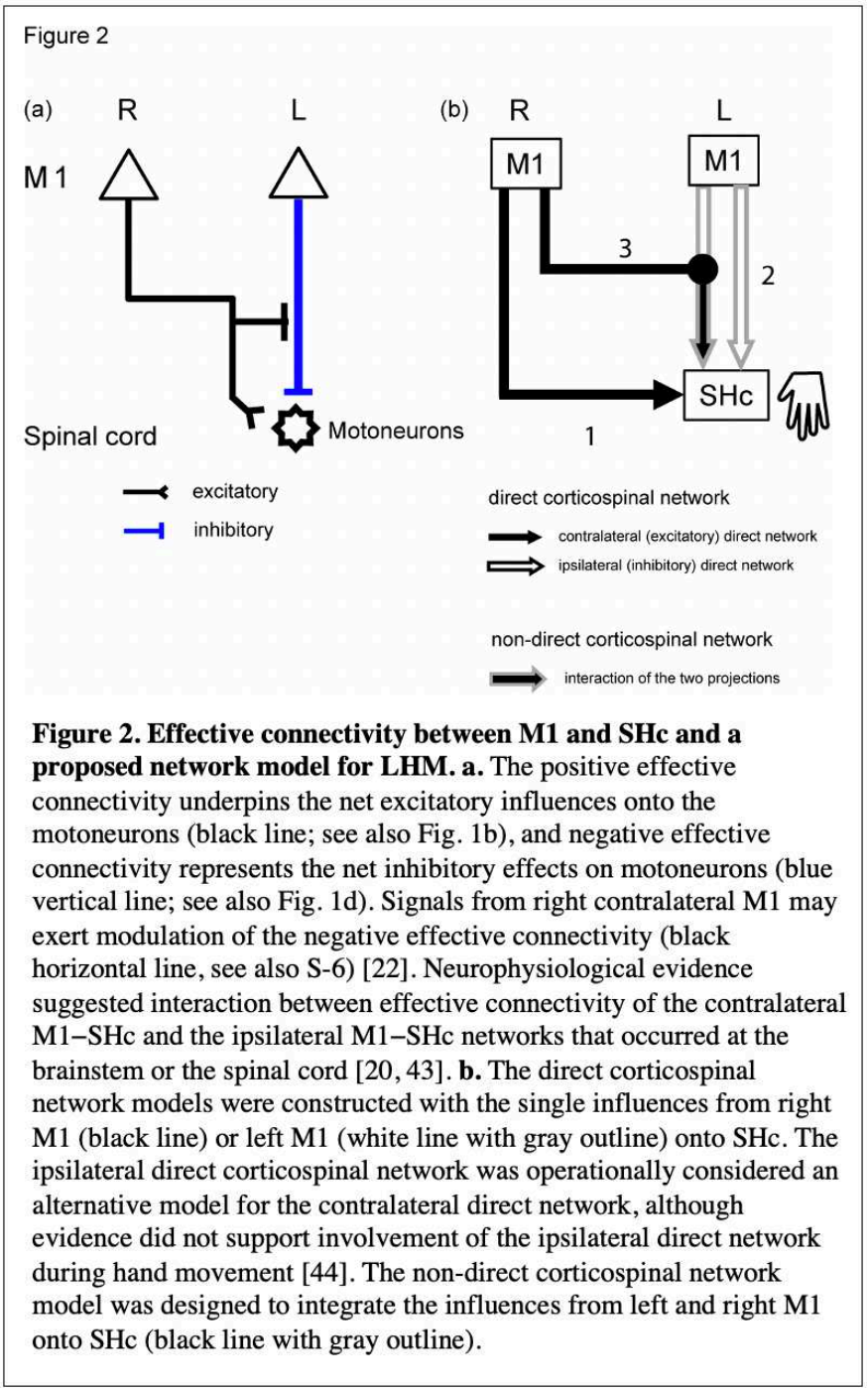

\section{Involvement of the non-direct corticospinal}

network pathway integrating the

contralateral and ipsilateral M1-SHc

\section{networks in LHM}

Our findings indicate that LHM involved a

more complicated network, consisting of the

contralateral M 1 -SHc and ipsilateral M1-

SHc networks, than did RHM. Anatomical

evidence supports the non-direct corticospinal

network connecting between the two M1-SHc

networks ${ }^{21}$. We thus used network modelling

to test the assumption that information from

both M1 hemispheres would be integrated to

activate ipsilateral SHc during LHM (Fig. 2).

Two possible forms of interactions between

the contralateral M1-SHc and the ipsilateral

M1-SHc networks influencing SHc activity were tested separately according to network model theories:

an interaction of "activity" between bilateral M1 or an interaction of the "connectivity" of the two M1

networks. First, we tested the activity model in which the non-direct corticospinal network might

integrate the effects of M1 activity onto ipsilateral SHc, but did not identify any models that explained 
SHc activity ( $p>0.2$ for all models, see $\mathrm{S}-8)$.

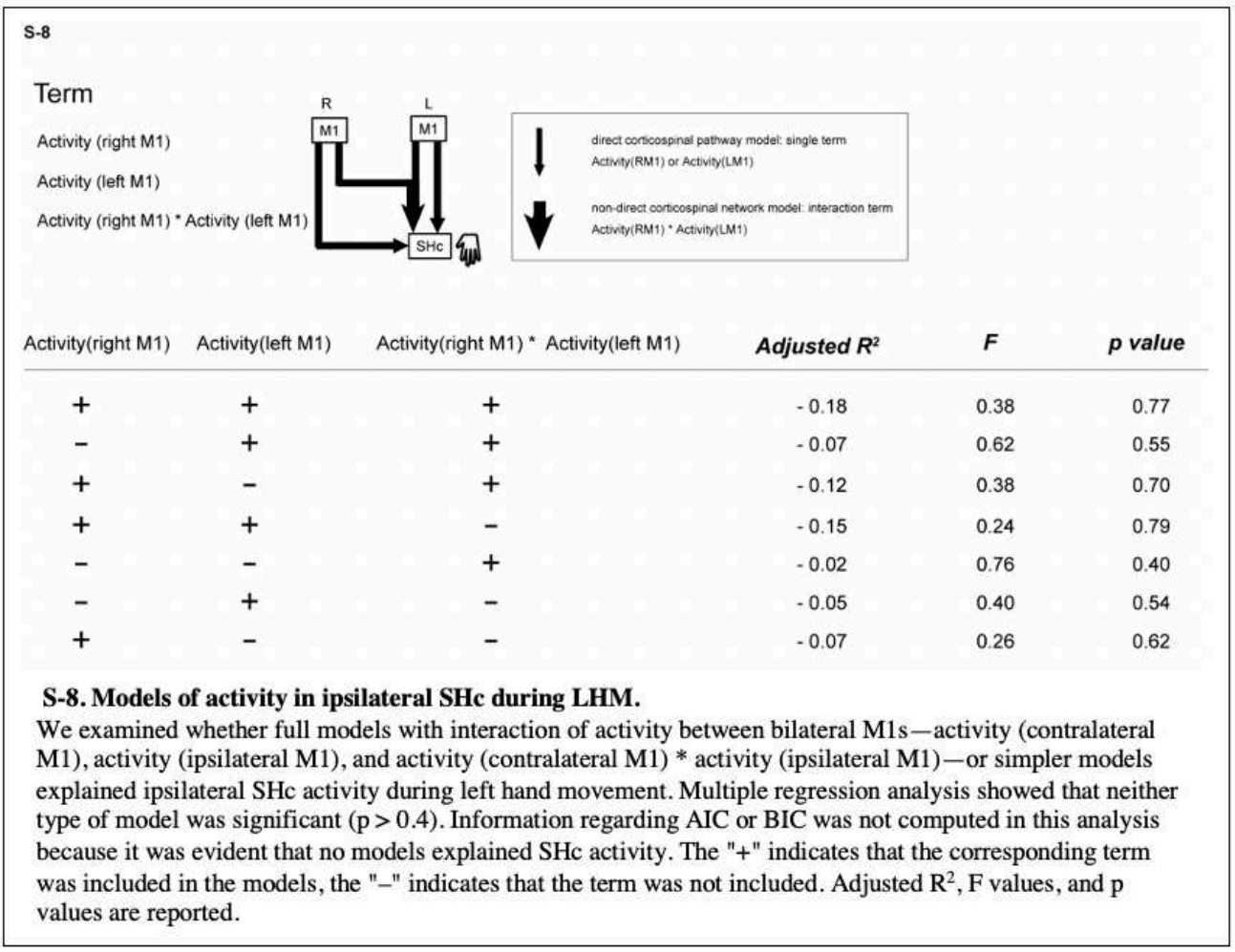

Next, we tested the connectivity model. According to the computational model referred to as structural equation modelling, the net connectivity of the non-direct corticospinal network was modelled as the 


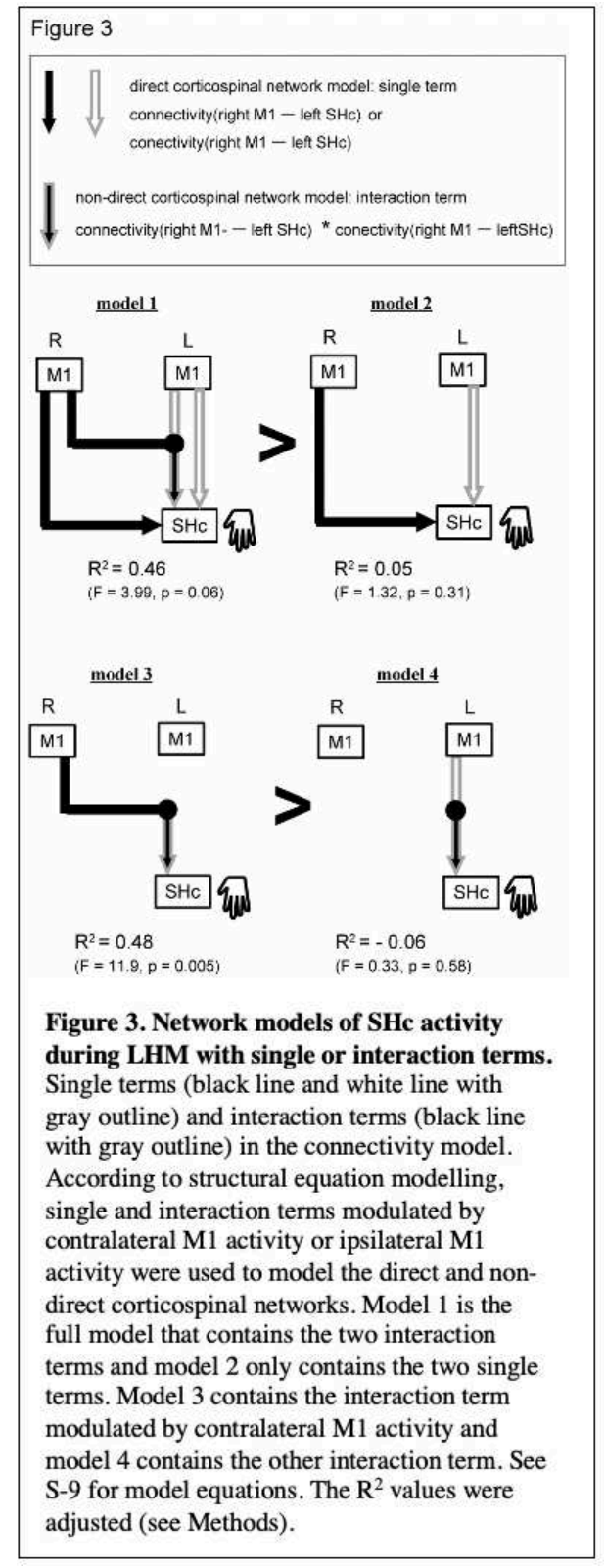

interaction of the connectivity of the contralateral and ipsilateral M1-SHc network ${ }^{29,30}$. The interaction term represents the mathematical multiplication of the connectivity of the two networks since they connect to each other. The interaction terms were designed to be modulated by either contralateral M1 activity or by ipsilateral M1 activity. The effects of the direct corticospinal network and those of the non-direct corticospinal network were separately tested in the full model. We compared the network models (Fig. 3), using adjusted $\mathrm{R}^{2}$ values along with Akaike information criterion and Bayesian information criterion (see Methods). The full network model with the two interaction terms (Fig. 3, model 1; S-9, equation $a$ ) was not significant $\left(\mathrm{R}^{2}\right.$ $=0.46, \mathrm{p}=0.06)$, but seemed to explain SHc activity better than the model with both direct corticospinal networks only $\left(\mathrm{R}^{2}=\right.$ $0.06, \mathrm{p}=0.31)($ model 2 , equation $f)$. Together, these results indicate that the interaction terms corresponding to the nondirect corticospinal network reasonably explain SHc activity. Next, we determined which of the interaction terms - the one modulated by contralateral M1 activity (model 3, equation $j$ ) or the other modulated by ipsilateral M1 activity (model 4, equation $l$ )—was the more important component in explaining SHc activity. This model, which only consisted of the interaction term modulated by contralateral M1 activity $\left(\mathrm{R}^{2}=0.48, \mathrm{p}=0.005\right)$, better explained the data variance than the model with the interaction term including only ipsilateral $\mathrm{M} 1$ activity $\left(\mathrm{R}^{2}=0.06, \mathrm{p}=0.58\right.$. It is important to note here that contralateral M1 can modulate SHc activity effectively by increasing effective connectivity of the ipsilateral M1-SHc network embedded in the non-direct corticospinal network (Fig. 3). These data 
support the fact that contralateral, rather than ipsilateral, M1 was a stronger source of modulation in SHc activity through the circuit integration of information from bilateral M1.

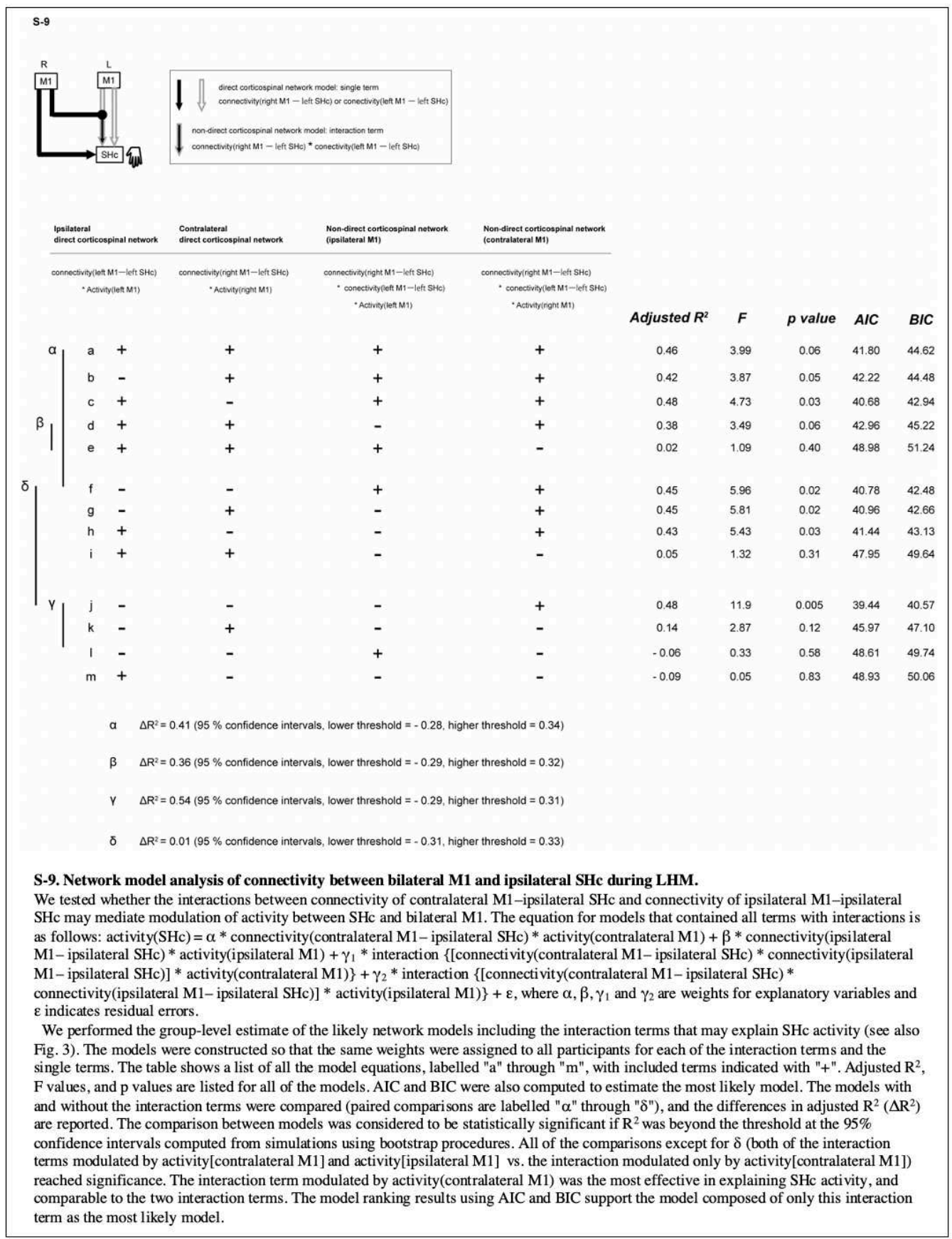

Individual differences in the involvement of the direct and non-direct corticospinal networks

\section{paralleling intersubject variability in the lateralization of hand preference}

Since the results of a previous study pointed to the functional specialization of the contralateral direct corticospinal network for preferred hand movement that reflected the lateralization of hand preference ${ }^{31}$, 
we hypothesized that the interindividual variability in the involvement of the direct and non-direct corticospinal networks may be correlated with that of the degree of preference for each hand. To test this, we first performed an individual level simulation of the involvement of each corticospinal network during either hand movement. Based on the network modelling analysis, the model included the two direct corticospinal networks and the non-direct corticospinal network modulated by contralateral M1 (Fig. 4).

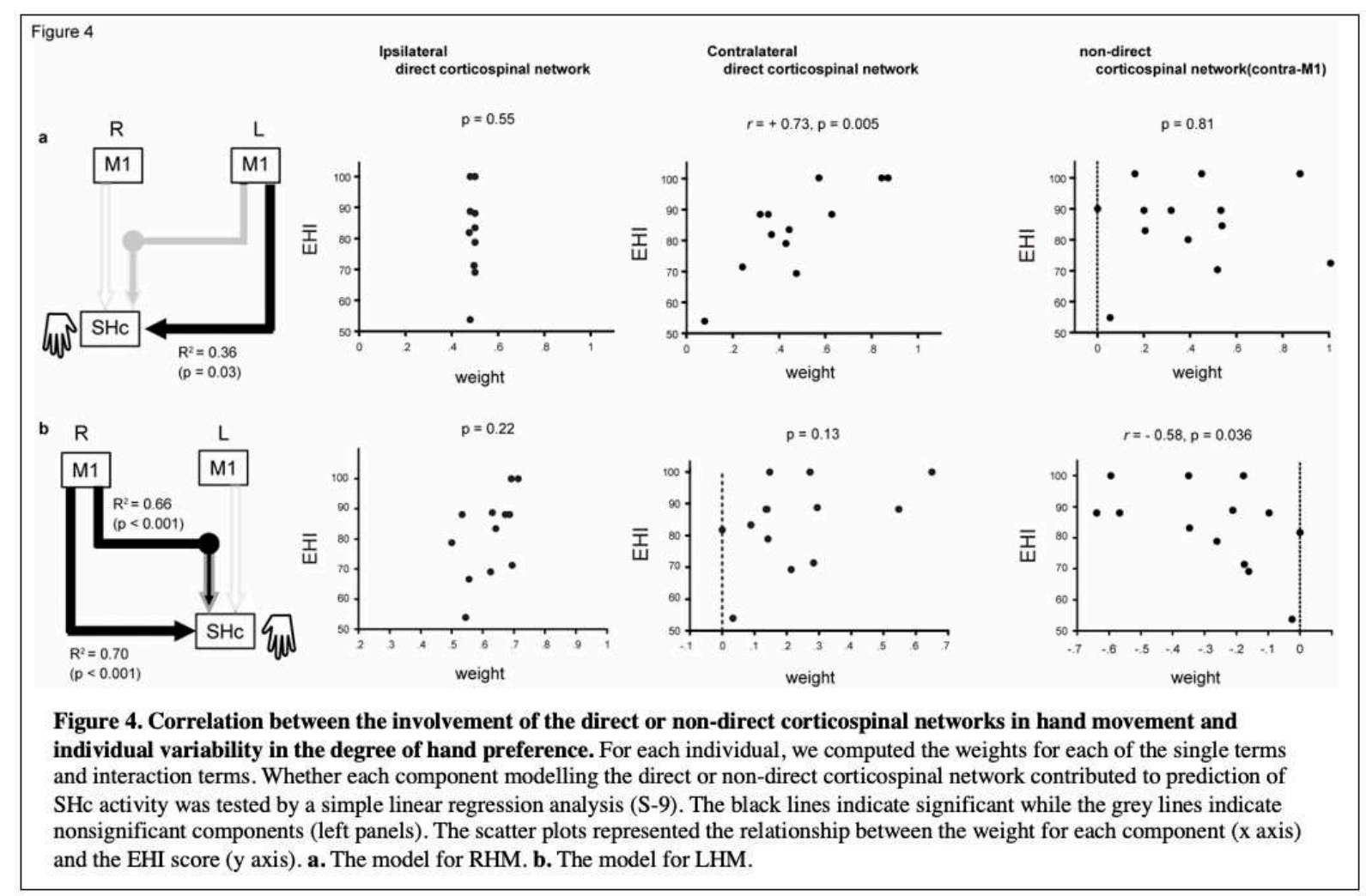

The weight for each component, indicating the degree to which each component might explain SHc activity, was optimized for each participant. During RHM, the component modelling the contralateral direct corticospinal network could predict SHc activity using the optimized weights $\left(\mathrm{R}^{2}=0.34, \mathrm{p}=0.03\right)$, while the other two components could not (S-10). During LHM, the components modelling the non-direct corticospinal network $\left(\mathrm{R}^{2}=0.66, \mathrm{p}<0.001\right)$ or the contralateral direct corticospinal network $\left(\mathrm{R}^{2}=0.70, \mathrm{p}\right.$ $<0.001$ ), but not those modelling the ipsilateral direct corticospinal network, could predict SHc activity 
using the optimized weights (Fig. 4 and S-10).

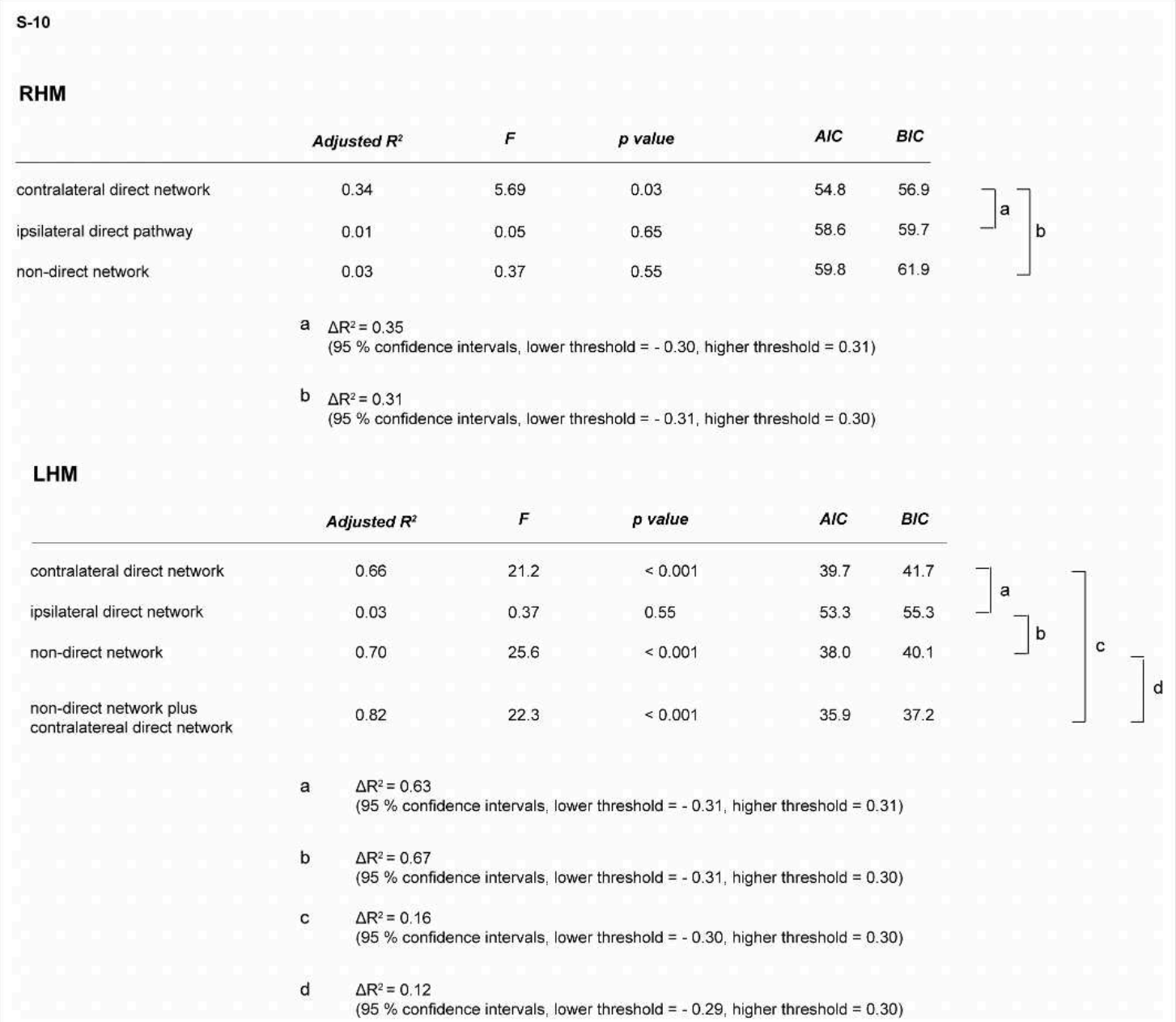

S-10. Simulation analysis of SHc activity during hand movement.

The simulation analysis was performed to estimate the individual participants' differences in the involvement of the direct and non-direct corticospinal networks. Our previous analyses (see S-6) suggested that the non-direct corticospinal network model modulated by contralateral M1 activity was the most likely one for LHM. It is also possible that the contralateral or ipsilateral direct corticospinal network models might participate in controlling LHM (see Fig. 2). Hence, those three components were all included in the model here. We used the same model for both right hand movement (RHM) and LHM. The equation is as follows: activity(SHc) $=\alpha *$ connectivity(contralateral M1-ipsilateral SHc) * activity (contralateral M1) $+\beta *$ connectivity(ipsilateral M1-ipsilateral SHc) * activity (ipsilateral M1) $+\gamma_{1} *$ interaction

$\{[$ connectivity(contralateral M1-ipsilateral SHc) $*$ connectivity(ipsilateral -M1-ipsilateral SHc) $] *$ activity $($ contralateral M1) $\}+\varepsilon$. The weights for each term were determined so that the residual errors were minimized for each individual in the model. Computed information regarding adjusted $\mathrm{R}^{2}, \mathrm{~F}$ values, $\mathrm{p}$ values, and $\mathrm{AIC}$ or BIC is listed for all models.

First, we tested if the components could predict ipsilateral SHc activity with use of the individually optimized weights for RHM or LHM. The component for the contralateral direct corticospinal network $\left(\mathrm{R}^{2}=0.34, \mathrm{p}=0.03\right)$ contributed to prediction of ipsilateral SHc activity in RHM more than that for the ipsilateral direct corticospinal network $\left(R^{2}=0.01, p=0.65\right)$ or the non-direct corticospinal network $\left(R^{2}=0.03\right.$, $p$ $=0.55)$. In the model for LHM, the component for the contralateral direct corticospinal network $\left(R^{2}=0.66, p=0.00075\right)$ or the non-direct corticospinal network $\left(R^{2}=0.70, p=0.00034\right)$ contributed to prediction of SHc activity, but the component for the ipsilateral direct corticospinal network did not $\left(\mathrm{R}^{2}=0.03, \mathrm{p}=0.58\right)$. There was no evidence that prediction accuracy was higher in the combination of the two components for the contralateral direct corticospinal network plus the non-direct corticospinal network $\left(R^{2}=0.817, p=0.00020\right)$ than the single component for only the non-direct corticospinal network or the contralateral direct corticospinal network. AIC and BIC estimated that the combination model was the most likely one.

Of note, an additional analysis (S-11) demonstrated that the contralateral direct corticospinal network was

less recruited during LHM than during RHM. Together, these data converge to point to the functionally

lateralized organization of the direct and non-direct corticospinal motor networks for RHM and LHM, 


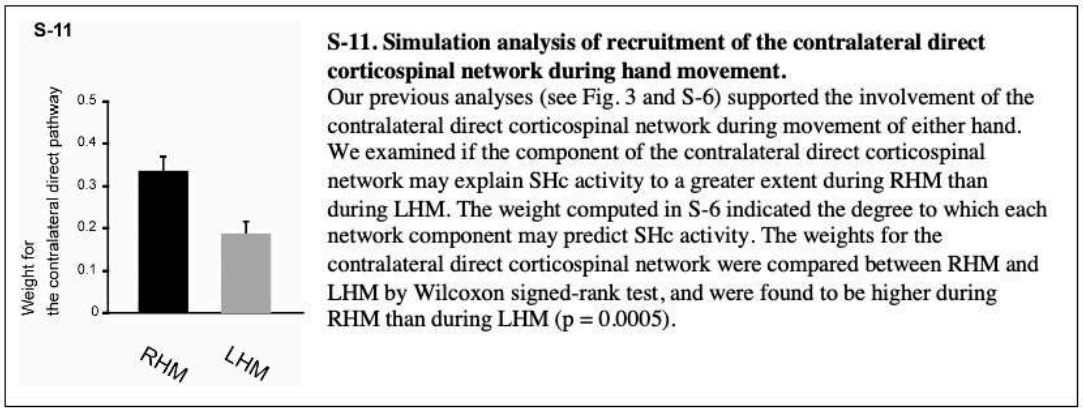

which we thereby further

assessed. The degree of

preference for right hand was

estimated using the Edinburgh

Handedness Inventory (EHI)

questionnaire. During RHM, a higher EHI score was correlated with a greater weight for the term

modelling the contralateral direct corticospinal network $(r=+0.58, \mathrm{p}=0.005$; Fig. $4 \mathrm{a})$. The other weights were not correlated with the EHI score during RHM. During LHM, a higher EHI score was correlated with a greater weight on the interaction term $(r=-0.58, \mathrm{p}=0.04$; Fig. $4 \mathrm{~b})$. The other weights were not correlated with the EHI score during LHM. These results indicate that the involvement of the direct corticospinal network in RHM is correlated with an individual's preference for the right hand. Notably, the involvement of the non-direct corticospinal network in nonpreferred LHM paralleled the lateralization of hand preference. Lastly, we confirmed that the interhemispheric connectivity between bilateral M1, or bilateral M1 activity itself, was not associated with hand preference (S-12).

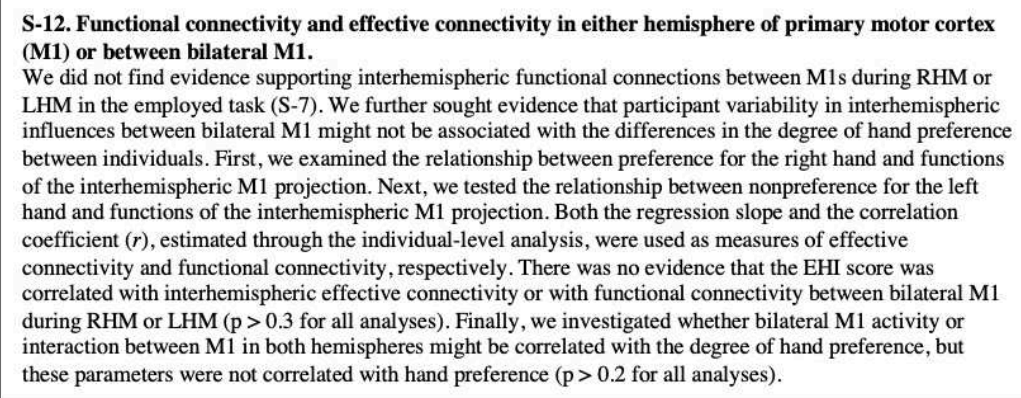

\section{Discussion}

Recent advances in MRI techniques have enabled the simultaneous measurement of activity in the brain and spinal cord, facilitating research that investigates functional interactions underlying the top-down motor control or sensory processing ${ }^{32-35}$. However, these studies did not address the relationship between the organization of the brain-spinal networks and daily behaviours in humans, such as hand preference.

Using corticospinal fMRI data, we were able to estimate recruitment of the direct and non-direct 
corticospinal networks that modelled monosynaptic or polysynaptic influences from M1s to motoneurons in the spinal cord. An effective connectivity analysis revealed the right-left asymmetric organization of the two corticospinal networks (Fig. 4 and S-10). We were able to link this finding to individual hand preference in daily activities. Using non-invasive techniques, our results have provided novel insights into the relationship between the functionally lateralized motor nervous system and behavioral asymmetry of handedness in humans. Noteworthy, the present results also suggested the human unique model where the phylogenetically new model of the direct corticospinal network preferably served the preferred hand movement while the old model of the non-direct corticospinal network participated in nonpreferred hand movement. Uniquely among primates, humans exhibit a strong bias in the functionally lateralized motor corticospinal pathways ${ }^{36}$, although no established theory has yet explained the biological mechanisms underlying this preference. Nevertheless, recent studies have provided hints with regard to the asymmetric organization of the human motor nervous system, even at prenatal stages. For example, fetuses already exhibit lateralized arm movements at 10 weeks' gestation ${ }^{37}$, despite fiber tracts connecting between the forebrain and the spinal cord have not been established yet at this stage ${ }^{38}$. Gene expression profiles have indicated maturation of the spinal cord at earlier weeks postconception than that of the forebrain ${ }^{4,5}$. Moreover, maturation pattern of the neural architectures differs between right and left SHc; namely, left SHc matures earlier than does right SHc. It is possible that the genetically programmed staggered maturation of the spinal cord triggers the asymmetrical organization of the anatomical projections between M1s and SHc that underpin the direct and non-direct corticospinal networks for controlling unilateral hand movement.

Evidence indicated the two computational networks that modelled monosynaptic or polysynaptic influences from M1s to motoneurons in the spinal cord ${ }^{2,3}$. Our results have first provided the neurocomputational model suggesting asymmetric organization of the two corticospinal networks underlying RHM and LHM in right-handed humans. Indeed, neurophysiological and imaging studies partly supported our results in right-handed healthy participants ${ }^{6,7,39}$; RHM or LHM involved contralateral M1, while LHM but not RHM involved ipsilateral M1; Integration of signals from bilateral 
M1s was observed during LHM but not RHM $^{8}$, and likely occurred at the brainstem or the spinal cord ${ }^{20}$. These lines of evidence corroborated the functionally lateralized direct and non-direct corticospinal networks in right-handed humans.

Our results provided a biological model that organization of the corticospinal motor networks related to a behavioural laterality of hand-use preference. Recent evidence indicates that ensemble patterns of activity in corticomotoneuronal M1 cells encode separate dexterous hand movements ${ }^{40}$. Transcranial magnetic stimulation over M1 showed a lower motor threshold for inducing muscle activation in intrinsic hand muscles when stimulating to left M1 than when stimulating to right $\mathrm{M} 1{ }^{41}$. Asymmetry in motor threshold between M1s in bilateral hemispheres was not found when motor threshold for proximal arm muscles was examined. Furthermore, a lower motor threshold for hand muscles in left M1 was correlated with a higher degree of the right-handedness assessed by Edinburgh handedness inventory ${ }^{41}$. It is likely that left M1 had a direct corticospinal connection to a greater number of motoneurons innervating to hand muscles than right $\mathrm{M} 1{ }^{41}$. Our results also supported that RHM involved the direct corticospinal network - that modelled the corticomotoneuronal connection - more than LHM (S-11). It is possible that use-dependent establishment of the corticomotoneuronal connection from left M1 to right SHc at postnatal term ${ }^{38}$ might be associated with preferable recruitment of the direct corticospinal network during preferred RHM in right-handedness humans. No previous studies addressed whether asymmetric involvement of the nondirect corticospinal network related to lateralization of hand preference. Our results suggested the model that nonpreferred hand movement preferably involved the non-direct corticospinal network (Fig. 4 and S10), although recruited the direct corticospinal network either. Several lines of evidence have implicated maturation of the non-direct corticospinal network at the prenatal term ${ }^{42}$ that probably occurs earlier than establishment of the direct corticospinal network ${ }^{38}$. It is thus possible that early-maturing left SHc motoneurons may receive stronger innervation from the non-direct corticospinal network than from the direct corticospinal network ${ }^{5}$. Observation in non-human primates revealed that the non-direct corticospinal network was less able to perform dexterous hand movement than the direct corticospinal 
network ${ }^{1}$. It seems plausible that the non-direct corticospinal network participated in nonpreferred LHM. This idea is consistent with the theory that "ontogeny recapitulates phylogeny" ${ }^{36}$.

In conclusion, this corticospinal fMRI study presented here has suggested the network model representing the asymmetric organization of the corticospinal networks for unilateral hand movement that underlie the lateralization of hand preference. We speculate that the developmental asymmetry of SHc maturation may trigger the lateralization of the corticospinal motor system, which may influence the asymmetric organization of the cerebral hemispheres ${ }^{5}$. This study opens a new avenue of research to explore how the lateralized corticospinal motor system which underlies the unique human trait of handedness may have triggered the acquisition of additional evolutionary gains in human beings over other primates. 


\section{Methods}

\section{Participants and study design}

we recruited 16 right-handed, healthy young adults with mean age of 21.4 years (range 18-26 years; 7 males, 9 females). None of the participants reported any history of neuropsychiatric disorders. Three participants were excluded after we found severe artefacts in their imaging data (see Preprocessing). The data from the remaining 13 participants ( 5 males, 8 females) were analysed and reported. The 13 participants were judged as right handed using the EHI ${ }^{10}$, by which the mean laterality quotient and standard deviation was $84.0 \pm 13.4$ (range 53.8-100). The participants were informed about the experimental procedure, and all of them participated in the experiment after giving written informed consent according to the study protocol approved by the National Center of Neurology and Psychiatry ethics committee.

In this experiment, the participants were required to gaze at the fixation point on a monitor, and to perform repetition of tapping with the thumb and small finger of either the right or left hand after presentation of an auditory cue every second (i.e., $1 \mathrm{~Hz}$; Presentation software, Neurobehavioral Systems, Albany, CA, USA). The task took $11 \mathrm{~s}$, and alternated between RHM and LHM. The task blocks were interleaved with resting blocks of $28.6 \mathrm{~s}$ each, in which participants were instructed not to move the fingers of either hand, although auditory cues were paced at the same frequency (i.e., $1 \mathrm{~Hz}$ ) as during the task. In total, the entire experiment consisted of 8 blocks for the task and 9 blocks of rest. Before beginning the experiment, participants practiced the finger tapping outside the magnetic resonance imaging (MRI) scanner to ensure that were familiar enough with the task to perform it well in the scanner.

In the MRI scanner, the participants' arms were aligned with the sides of their body and 
their hands were placed in a supine position. The bilateral upper limbs and the bodies of the participants were fixed to the scanner bed with band restraints to minimize joint and neck movements during the finger tapping task. Participants were also instructed to relax and remain still in order to minimize motion artefacts and imaging noise at the level of the cervical spinal cord.

To ensure that that the participants performed the task using the assigned hand without mirror movements with the opposite hand, electoromyography (EMG) signals were visually observed in real time from the bilateral muscles of the deltoid, the brachialis and brachioradialis, the abductor policis brevis, and the abductor digiti minimi and recorded using BrainAmp ExG MR (Brain Products, Gilching, Germany). Surface electrodes with shielded plates and cables were placed over the muscles with an interelectrode distance of approximately $2 \mathrm{~cm}$, and a ground electrode was placed on the dorsal surface of the right wrist.

\section{Data Acquisition}

We used a 3 Tesla MAGNETOM Verio MRI scanner (Siemens, Erlangen, Germany) with standard 12 channel head coil and 4 channel neck array coil to measure signal changes in regions of interest including M1 in both hemispheres and the cervical spinal cord. For simultaneous scanning of the two distant areas in one single volume, we applied acquisition of multiple slices along a sagittal plane covering from top of the head to the upper thoracic spinal cord at the segmental level of Th1 along a rostro-caudal axis. We acquired the BOLD sensitive, gradient-echo, echo planar imaging (EPI) sequence combined with Generalized Autocalibrating Partially Parallel Acquisition. EPI parameters were as follows: repetition time $=2600 \mathrm{~ms}$; echo time $=25 \mathrm{~ms}$; flip angle $=75$ degrees; acceleration factor for GRAPPA $=2$; rectangular field of 
view, 190 (anterior-posterior) $\times 320$ (rostral-caudal) $\mathrm{mm}$; matrix size $=$ in-plane resolution of 2.5 (anterior-posterior) $\times 2.5$ (rostral-caudal) $\mathrm{mm}^{2}$; slice thickness $=3 \mathrm{~mm}$ (left-right) and 44 slices. S-1 shows an example of the functional image acquired with the present imaging protocol.

\section{Functional MRI data analysis}

\section{Preprocessing}

We collected data from 16 participants but excluded three participants for the following reasons: severe $\mathrm{N} / 2$ ghosting $(\mathrm{n}=2)$ and severe distortion artefact in the spinal cord $(\mathrm{n}=1)$. Thus, the results were obtained from the remaining 13 participants ( 5 males, 8 females). The functional MRI (fMRI) data were preprocessed using the free distribution software SPM8 (http://www.fil.ion.ucl.ac.uk/spm/) and FSL (https://fsl.fmrib.ox.ac.uk/fsl/fslwiki/FSL). The first eight volumes of fMRI run were discarded to allow the MRI signal to reach T1 equilibrium. Preprocessing steps included motion correction and slice timing correction to the first slice.

Correction for residual motion was performed for all functional images using the SPM standard six parameter rigid body transformation. As compared with the brain fMRI data, spinal fMRI is much more susceptible to artefacts derived from the cerebrospinal fluid (CSF) and other biological factors. This increased susceptibility in spinal fMRI can be explained by the small cross-sectional cord area where fMRI signals can be contaminated by faint motion and by pulsation of surrounding CSF; magnetic field inhomogeneity causing distortion and ghosting artefacts in the encoding direction; and physiological noises due to respiration and cardiac beats masking BOLD signals in the spinal cord ${ }^{43-46}$. Therefore, to detect BOLD signals in the spinal cord, it is ideal to remove as much physiological noise as possible ${ }^{43-45}$. To do this, we used the independent component analysis in FSL (Multivariate Exploratory Linear Optimized 
Decomposition into Independent Components) for decomposing the components corresponding to noise and artefacts 47,48 . Through this procedure we removed biological noise and corrected several artefacts related to motion and CSF pulsation ${ }^{49}$.

Most fMRI studies have focused on brain activity and the required spatial normalization of an individual's brain images to the anatomical standard template to localize regional activity for analysis of group data. For the brain imaging data, fMRI images were spatially normalized to fit the Montreal Neurological Institute template based on the standard stereotaxic coordinate system to obtain the group level coordinates of M1 and other areas. All images were smoothed with an isotropic Gaussian kernel of $4 \mathrm{~mm}$ full width at half maximum. Low frequency noise was removed with high-pass filter, and serial correlations were adjusted using an autoregressive model.

However, there is no consensus yet for the methodology to spatially normalize individual images from spinal cord scans, although previous studies have attempted to develop a methodology $32,33,46$. Therefore, we decided not to apply spatial normalization, and instead estimated spinal activity on the individual's anatomical space. It is widely accepted from surgical exploration that the segment of the spinal cord at C7 and Th1 - the region of interest activated by the finger tapping task - is located at the same level of the vertebral bodies of C6 and C7 ${ }^{50}$. Thus, for determining its anatomical localization, we referred to the spinal segments as the anatomical landmarks.

$\underline{\text { Voxel-wise analysis }}$

We performed voxel-wise analysis to search for activated voxels in bilateral M1 and SHc. Vectors representing the experimental paradigm were modelled for the individual-level general 
linear model analysis through convolution of a boxcar function (task duration: $28.8 \mathrm{~s}$ ) with the canonical hemodynamic response function. Six parameters ( 3 translations and 3 rotations) representing motion of the head and spinal cord were included in the design matrix as covariates of no interest. Then we tested main effects of hand movement (i.e., RHM or LHM relative to the rest period) in each participant.

Activation in the spinal cord was examined in individual level analysis (see

Preprocessing). The threshold was set at uncorrected $\mathrm{p}=0.05$ using a one sample t-test [17]. The volume of interest (VOI) for SHc was defined as the activated voxels in the anatomical template for each participant (see VOI analysis). Activation in bilateral M1was tested in group level analysis after spatial normalization of the individual participants' fMRI data. The individual-level contrast images were obtained from each participant and then used for the group-level analysis. The group-analysis was done using a one sample t-test. The family-wise error (FWE) corrected threshold was set at $p=0.05$ with use of the small volume correction (SVC) method ${ }^{16}$.

\section{$\underline{\text { VOI analysis }}$}

To investigate task evoked activation in the spinal cord, we computed beta estimate values from the individual level analysis and extracted individuals' BOLD time course data within activated voxels in bilateral M1 and SHc. The VOIs of the spinal segments C7-Th1 and C5 were drawn manually by hand in each participant by referring to the individual's coregistered anatomical T1 image. The VOIs were separated in the midline between the right and left hemispheres. The $\beta$ values were averaged among voxels within the VOIs for RHM and LHM, and then averaged across participants. Three-way repeated measures ANOVA with factors "hand" (RHM/LHM), "segment" (C5/C7-Th1) and "side" (right/left) on $\beta$ values were examined 
(see Fig. 1) for stronger activity in the C7-Th1 compared with the C5 segment (i.e., "segment" effect), and also for stronger activity in the hemisphere ipsilateral to the moving hand compared with the contralateral hemisphere (i.e., "side" effect). Posthoc comparisons were also done using the Holm-Bonferroni method ${ }^{51}$. The time series data was used for effective connectivity and functional connectivity analyses (see Fig. 1, S-4, S-5 and S-7). To improve the signal-to-noise ratio in the spinal activity data, a dataset of the mean of 11 time points was computed by averaging each time point across four blocks of each hand movement in each individual.

To estimate bilateral M1 activity, the beta estimate values and time series data for individuals were computed in their VOIs in the same way as described above. The only difference was that the VOIs were created on the standard anatomical template. The group level, voxel-wise analysis was performed. We searched for the peak coordinate of M1 activity inside

Brodmann area 4 based on the anatomical atlas (http://fmri.wfubmc.edu/software/pickatlas), with a FWE corrected threshold of $\mathrm{p}<0.05$. The three dimensional, sphere shaped VOIs were created with a $8 \mathrm{~mm}$ radius centred at its peak coordinate for the right or left M1 in each participant. The estimate beta values were examined for replication of bilateral M1 activity during RHM or LHM (S-3). The dataset of the mean of 11 time points with respect to bilateral M1 was computed in the same way as described above.

\section{Effective connectivity analysis and functional connectivity analysis}

Effective connectivity and functional connectivity analyses were performed using a paired dataset of the mean of 11 time points with respect to activity in bilateral M1 and SHc. Because variance differed between regions, time series data for an individual participant was transformed to z scores in each region for both connectivity analyses. Note that raw, 
nontransformed data of SHc activity and M1 activity is depicted in Figure 1, S-2, and S-3 for display purposes. In the effective connectivity analysis, single linear regression analysis was performed to compute the value of $\mathrm{R}^{2}$ and the regression slope in each participant using the time series data in M1 and SHc during hand movement and rest. We identified the task dependent change in effective connectivity by comparing regression slopes between hand movement and rest within participants. In the functional connectivity analysis, correlation analysis was performed using the same time series dataset in the two regions during RHM or LHM and rest in each participant. To estimate presence of the functional connections relevant to the task, the correlation coefficient $(r)$ was compared between hand movement and rest within participants. The within subject comparison for the functional and effective connectivity analyses was made using the Wilcoxon signed-rank test.

\section{Network model analysis}

Regression analyses were used to examine how interaction of the influences derived from contralateral and ipsil-M1 might determine the net influences between bilateral M1 and the spinal cord. We hypothesized two forms of interactions. First, as in previous studies ${ }^{19}$, we examined how interaction of activity in bilateral M1 might affect activity in SHc ipsilateral to the moving hand (see S-8 and refer to the equation corresponding to the interaction model). Next, interaction of connectivity term was tested to examine how integration of connectivity of the contralateral M1-SHc and ipsilateral M1-SHc networks might determine net connectivity between bilateral M1 and SHc (see S-9 and refer to the equations corresponding to the interaction models). The non-direct corticospinal network model consisted of the interaction terms modulated by bilateral M1 activity: activity(ipsilateral $\mathrm{SHc})=\alpha *$ interaction $\{[$ connectivity (contralateral M1-SHc) * connectivity(ipsilateral M1-SHc)] 
activity $($ contralateral M1) $\}+\beta *$ interaction $\{[$ connectivity $($ contralateral $\mathrm{M} 1-\mathrm{SHc}) *$ connectivity(ipsilateral M1-SHc)] * activity(ipsilateral M1) $\}+\varepsilon$, where $\alpha$ and $\beta$ were terms for explanatory variables and $\varepsilon$ indicated residual errors. Since models with the two components of the interaction terms were found to be significant, further comparisons between the components were made. Since the number of terms differed between models, the adjusted $\mathrm{R}^{2}$ was computed. The bootstrap procedure was used to statistically compare models. The null distributions were created by the following simulation methods using the original dataset: choosing arbitrary participants, swapping the data with respect to the activity and slope separately within the chosen participants and then computing the value of $\mathrm{R}^{2}$, and the slope on multiple regression analysis based on the simulated data. Significance was set to values beyond $95 \%$ confidence intervals estimated from the simulated null distributions.

The simulation analysis was applied to the selected network model for RHM and LHM in order to estimate an individual's differences in the involvement of the direct and non-direct corticospinal networks (Fig. 7 and S-6). We predicted that the involvement of the direct and nondirect corticospinal networks might be associated with preferred right hand and nonpreferred left hand, respectively. The weight for each term was optimized so that residual errors were minimized for each individual in the model. The constraints for the weights were set on the basis of the results acquired from the group level network model analysis (S-6): the weights for the contralateral and ipsilateral direct corticospinal network model were positive (i.e., more than zero). The weight was negative for the non-direct corticospinal network model (i.e., less than zero). Values of the weights for each individual are shown in Figure 7. We investigated the correlation between the weights of the direct or non-direct corticospinal networks and the degree of hand preference. Hand preference was estimated by the EHI questionnaire. The values of their 
weights and the EHI scores were used in the correlation analysis.

\section{Correlation analysis}

We used correlation analyses to compute the correlation coefficient $(r)$ and its statistical significance (Fig. 3, Fig. 4, and S-2 to S-4; details for each analysis are in the main text, figure legends, and supplementary data). The M1 activity and SHc activity were transformed to zscores with respect to each variable and then tested. The significance threshold was set at $\mathrm{p}<$ 0.05 .

\section{Statistical information}

All analyses used data from all 13 participants. A two-tailed test was used in all of the analyses: either the Wilcoxon signed-rank test or Pearson's correlation coefficient (Fig. 3, Fig.4, and S-2 to S-4, and S-9). The highest and lowest 95\% confidence interval was determined from the simulated null distributions. F values, $t$ values, and the degree of freedom for all results are provided in the figures.

Analyses in the present study were performed using the SPSS software package (IBM, Chicago, IL, USA), and the MATLAB Statistics and Machine Learning Toolbox 8.1 and Optimization Toolbox 8.1 (Release 2017b, The MathWorks, Inc., Natick, MA, USA).

\section{Data Availability}

The following pieces of data from individual participants are available online (https://rive.google.com/drive/u/0/folders/1dWuVpZ5ogrY3EVbUdgjeg5aqwNis3QFQ): the EHI index (Fig. 7), BOLD activity in bilateral M1 and SHc (Fig. 1, Fig. 2, and S-5), the regression 
slope obtained from regression analyses (i.e., effective connectivity; Fig. 3), and the correlation coefficient computed from correlation analyses (i.e., functional connectivity; S-2 to S-4). These data were the basis for the network analyses. The weights calculated in the network analyses

(Fig. 6, Fig. 7, and S-5 to S-7) are also provided online.

Code availability

We employed the corrcoef, regress, and fmincon MATLAB functions for the regression analyses, correlation analyses, and network analyses. The codes implementing the functions are provided from the corresponding authors upon request. 


\section{Acknowledgements}

The research was supported by the JSPS KAKENHI Grant, no. 959897 (T.H.); 16H01494, 16H01600 and 19H04449 (M.A.) and Grant of Japan Orthopaedics and Traumatology Research Foundation, Inc, No. 365 (E.T.). Author contributions were as follows: All authors helped conceive and design the experiment. E.T. and T.H. conducted an experiment. E.T. collected fMRI data. E.T. and M.A. performed effective/functional connectivity analysis. M.A. performed network model analyses. M.A. and T.H. wrote the manuscript.

\section{Author Contributions}

E.T., M.A. and T.H conducted a research. E.T. and M.A. analyzed data. M.A. performed network analyses. M.A. and T.H. wrote the manuscript. T.H. supervised this research.

\section{Competing interests}

The authors declare no competing interests. Correspondence and requests for materials should be addressed to M.A. (mitsunari.abe.a8@ncnp.go.jp) or T.H. (hanakawa.takashi2s@kyoto-u.ac.jp).

\section{List of Supplementary Materials}

Figs. S1-S12

References (49-58) 


\section{References}

1. Isa, T., Kinoshita, M. \& Nishimura, Y. Role of Direct vs. Indirect Pathways from the Motor Cortex to Spinal Motoneurons in the Control of Hand Dexterity. Front Neurol 4, 191 (2013).

2. Rathelot, J.A. \& Strick, P.L. Subdivisions of primary motor cortex based on cortico-motoneuronal cells. Proc Natl Acad Sci U S A 106, 918-923 (2009).

3. Witham, C.L., Fisher, K.M., Edgley, S.A. \& Baker, S.N. Corticospinal Inputs to Primate

Motoneurons Innervating the Forelimb from Two Divisions of Primary Motor Cortex and Area 3a. $J$

Neurosci 36, 2605-2616 (2016).

4. de Kovel, C.G.F., et al. Left-Right Asymmetry of Maturation Rates in Human Embryonic Neural Development. Biol Psychiatry 82, 204-212 (2017).

5. de Kovel, C.G.F., Lisgo, S.N., Fisher, S.E. \& Francks, C. Subtle left-right asymmetry of gene expression profiles in embryonic and foetal human brains. Sci Rep 8, 12606 (2018).

6 . Chen, R., Cohen, L.G. \& Hallett, M. Role of the ipsilateral motor cortex in voluntary movement. Can J Neurol Sci 24, 284-291 (1997).

7. Chen, R., Gerloff, C., Hallett, M. \& Cohen, L.G. Involvement of the ipsilateral motor cortex in finger movements of different complexities. Ann Neurol 41, 247-254 (1997).

8. van den Berg, F.E., Swinnen, S.P. \& Wenderoth, N. Involvement of the primary motor cortex in controlling movements executed with the ipsilateral hand differs between left- and right-handers. $J$ Cogn Neurosci 23, 3456-3469 (2011).

9. Bradnam, L.V., Stinear, C.M. \& Byblow, W.D. Ipsilateral motor pathways after stroke:

implications for non-invasive brain stimulation. Front Hum Neurosci 7, 184 (2013).

10. Oldfield, R.C. The assessment and analysis of handedness: the Edinburgh inventory.

Neuropsychologia 9, 97-113 (1971).

11. Maieron, M., et al. Functional responses in the human spinal cord during willed motor actions: evidence for side- and rate-dependent activity. J Neurosci 27, 4182-4190 (2007).

12. Ng, M.C., et al. Cervical spinal cord BOLD fMRI study: modulation of functional activation by dexterity of dominant and non-dominant hands. Neuroimage 39, 825-831 (2008).

13. Weber, K.A., 2nd, Chen, Y., Wang, X., Kahnt, T. \& Parrish, T.B. Lateralization of cervical spinal cord activity during an isometric upper extremity motor task with functional magnetic resonance imaging. Neuroimage 125, 233-243 (2016).

14. Yoshino-Saito, K., Nishimura, Y., Oishi, T. \& Isa, T. Quantitative inter-segmental and inter-laminar comparison of corticospinal projections from the forelimb area of the primary motor cortex of macaque monkeys. Neuroscience 171, 1164-1179 (2010).

15. Soteropoulos, D.S., Edgley, S.A. \& Baker, S.N. Spinal commissural connections to motoneurons controlling the primate hand and wrist. J Neurosci 33, 9614-9625 (2013).

16. Newton, J.M., Sunderland, A. \& Gowland, P.A. fMRI signal decreases in ipsilateral primary motor cortex during unilateral hand movements are related to duration and side of movement. Neuroimage

24, 1080-1087 (2005).

17. Hayashi, M.J., et al. Hemispheric asymmetry of frequency-dependent suppression in the ipsilateral primary motor cortex during finger movement: a functional magnetic resonance imaging study. Cereb Cortex 18, 2932-2940 (2008).

18. Tzourio-Mazoyer, N., et al. Between-hand difference in ipsilateral deactivation is associated with hand lateralization: fMRI mapping of 284 volunteers balanced for handedness. Front Hum Neurosci 9, 5 (2015).

19. Friston, K.J. Functional and effective connectivity: a review. Brain Connect 1, 13-36 (2011). 
20. Gerloff, C., et al. Inhibitory influence of the ipsilateral motor cortex on responses to stimulation of the human cortex and pyramidal tract. J Physiol 510 ( Pt 1), 249-259 (1998).

21. Alstermark, B., Isa, T., Pettersson, L.G. \& Sasaki, S. The C3-C4 propriospinal system in the cat and monkey: a spinal pre-motoneuronal centre for voluntary motor control. Acta Physiol (Oxf) 189, 123-140 (2007).

22. Isa, T., Ohki, Y., Seki, K. \& Alstermark, B. Properties of propriospinal neurons in the C3-C4 segments mediating disynaptic pyramidal excitation to forelimb motoneurons in the macaque monkey. $J$ Neurophysiol 95, 3674-3685 (2006).

23. Isa, T., Ohki, Y., Alstermark, B., Pettersson, L.G. \& Sasaki, S. Direct and indirect corticomotoneuronal pathways and control of hand/arm movements. Physiology (Bethesda) 22, 145-152 (2007).

24. Kobayashi, M., Hutchinson, S., Schlaug, G. \& Pascual-Leone, A. Ipsilateral motor cortex activation on functional magnetic resonance imaging during unilateral hand movements is related to interhemispheric interactions. Neuroimage 20, 2259-2270 (2003).

25. Etoh, S., et al. Stroke patient with mirror movement of the affected hand due to an ipsilateral motor pathway confirmed by transcranial magnetic stimulation: a case report. Int J Neurosci 120, 231235 (2010).

26. Perez, M.A. \& Cohen, L.G. Mechanisms underlying functional changes in the primary motor cortex ipsilateral to an active hand. J Neurosci 28, 5631-5640 (2008).

27. Grefkes, C., Eickhoff, S.B., Nowak, D.A., Dafotakis, M. \& Fink, G.R. Dynamic intra- and interhemispheric interactions during unilateral and bilateral hand movements assessed with $\mathrm{fMRI}$ and DCM. Neuroimage 41, 1382-1394 (2008).

28. Amann, M., Hirsch, J.G. \& Gass, A. A serial functional connectivity MRI study in healthy individuals assessing the variability of connectivity measures: reduced interhemispheric connectivity in the motor network during continuous performance. Magn Reson Imaging 27, 1347-1359 (2009).

29. Morgan, V.L., Abou-Khalil, B. \& Rogers, B.P. Evolution of functional connectivity of brain networks and their dynamic interaction in temporal lobe epilepsy. Brain Connect 5, 35-44 (2015).

30. Verhoeven, T., et al. Automated diagnosis of temporal lobe epilepsy in the absence of interictal spikes. Neuroimage Clin 17, 10-15 (2018).

31. Darling, W.G., et al. Laterality affects spontaneous recovery of contralateral hand motor function following motor cortex injury in rhesus monkeys. Exp Brain Res 228, 9-24 (2013).

32. Eippert, F., et al. Activation of the opioidergic descending pain control system underlies placebo analgesia. Neuron 63, 533-543 (2009).

33. Eippert, F., Finsterbusch, J., Bingel, U. \& Buchel, C. Direct evidence for spinal cord involvement in placebo analgesia. Science 326, 404 (2009).

34. Vahdat, S., et al. Simultaneous Brain-Cervical Cord fMRI Reveals Intrinsic Spinal Cord Plasticity during Motor Sequence Learning. PLoS Biol 13, e1002186 (2015).

35. Tinnermann, A., Geuter, S., Sprenger, C., Finsterbusch, J. \& Buchel, C. Interactions between brain and spinal cord mediate value effects in nocebo hyperalgesia. Science 358, 105-108 (2017).

36. Gunturkun, O. \& Ocklenburg, S. Ontogenesis of Lateralization. Neuron 94, 249-263 (2017).

37. Hepper, P.G. The developmental origins of laterality: fetal handedness. Dev Psychobiol 55, 588595 (2013).

38. Eyre, J.A., Miller, S., Clowry, G.J., Conway, E.A. \& Watts, C. Functional corticospinal projections are established prenatally in the human foetus permitting involvement in the development of spinal motor centres. Brain 123 ( Pt 1), 51-64 (2000).

39. van den Berg, F.E., Swinnen, S.P. \& Wenderoth, N. Excitability of the motor cortex ipsilateral to the moving body side depends on spatio-temporal task complexity and hemispheric specialization. PLoS One 6, e17742 (2011). 
40. Griffin, D.M., Hoffman, D.S. \& Strick, P.L. Corticomotoneuronal cells are "functionally tuned". Science 350, 667-670 (2015).

41. Macdonell, R.A., et al. Hemispheric threshold differences for motor evoked potentials produced by magnetic coil stimulation. Neurology 41, 1441-1444 (1991).

42. Bicknell, H.R., Jr. \& Beal, J.A. Axonal and dendritic development of substantia gelatinosa neurons in the lumbosacral spinal cord of the rat. J Comp Neurol 226, 508-522 (1984).

43. Giove, F., et al. Issues about the fMRI of the human spinal cord. Magn Reson Imaging 22, 15051516 (2004).

44. Kong, Y., Jenkinson, M., Andersson, J., Tracey, I. \& Brooks, J.C. Assessment of physiological noise modelling methods for functional imaging of the spinal cord. Neuroimage 60, 1538-1549 (2012).

45. Stroman, P.W., Lee, A.S., Pitchers, K.K. \& Andrew, R.D. Magnetic resonance imaging of neuronal and glial swelling as an indicator of function in cerebral tissue slices. Magn Reson Med 59, 700-706 (2008).

46. Stroman, P.W., et al. The current state-of-the-art of spinal cord imaging: methods. Neuroimage 84, 1070-1081 (2014).

47. Kelly, R.E., Jr., et al. Visual inspection of independent components: defining a procedure for artifact removal from fMRI data. J Neurosci Methods 189, 233-245 (2010).

48. Kelly, R.E., et al. Hybrid ICA-Seed-Based Methods for fMRI Functional Connectivity Assessment: A Feasibility Study. Int J Biomed Imaging 2010 (2010).

49. Shitara, H., Shinozaki, T., Takagishi, K., Honda, M. \& Hanakawa, T. Time course and spatial distribution of $\mathrm{fMRI}$ signal changes during single-pulse transcranial magnetic stimulation to the primary motor cortex. Neuroimage 56, 1469-1479 (2011).

50. Seichi, A., et al. Neurologic level diagnosis of cervical stenotic myelopathy. Spine (Phila Pa 1976) 31, 1338-1343 (2006).

51. Holm, S. A Simple Sequentially Rejective Multiple Test Procedure. Scandinavian Journal of Statistics 6, 65-70 (1979).

52. Riddle, C.N. \& Baker, S.N. Convergence of pyramidal and medial brain stem descending pathways onto macaque cervical spinal interneurons. J Neurophysiol 103, 2821-2832 (2010).

53. Soteropoulos, D.S., Edgley, S.A. \& Baker, S.N. Lack of evidence for direct corticospinal contributions to control of the ipsilateral forelimb in monkey. J Neurosci 31, 11208-11219 (2011). 


\section{Figure Legends}

\section{Figure 1. Asymmetric recruitment of the ipsilateral corticospinal network during hand}

movement.

a. Correlation between activity in contralateral M1 (x axis) and ipsilateral SHc (y axis) during RHM (left panel) or LHM (right panel) in a representative participant. Dots indicate the mean activity at each time point during hand movements. b. Effective connectivity (contralateral M1ipsilateral SHc) for RHM and LHM during the task and during rest periods. ${ }^{*} \mathrm{p}<0.001$. c. Correlation between ipsilateral M1 activity (x axis) and ipsilateral SHc activity (y axis) in the same representative participant. d. Effective connectivity (ipsilateral M1-ipsilateral SHc) for RHM and LHM during the task and rest periods $(* * \mathrm{p}=0.03, * * *$ indicates $\mathrm{p}=0.01)$. 
Figure 2. Effective connectivity between M1 and SHc and a proposed network model for

LHM. a. The positive effective connectivity underpins the net excitatory influences onto the motoneurons (black line; see also Fig. 1b), and negative effective connectivity represents the net inhibitory effects on motoneurons (blue vertical line; see also Fig. 1d). Signals from right contralateral M1 may exert modulation of the negative effective connectivity (black horizontal line, see also S-6) ${ }^{22}$. Neurophysiological evidence suggested interaction between effective connectivity of the contralateral M1-SHc and the ipsilateral M1-SHc networks that occurred at the brainstem or the spinal cord ${ }^{20,52} . \mathbf{b}$. The direct corticospinal network models were constructed with the single influences from right M1 (black line) or left M1 (white line with gray outline) onto SHc. The ipsilateral direct corticospinal network was operationally considered an alternative model for the contralateral direct network, although evidence did not support involvement of the ipsilateral direct network during hand movement ${ }^{53}$. The non-direct corticospinal network model was designed to integrate the influences from left and right M1 onto SHc (black line with gray outline). 
Figure 3. Network models of SHc activity during LHM with single or interaction terms.

Single terms (black line and white line with gray outline) and interaction terms (black line with gray outline) in the connectivity model. According to structural equation modelling, single and interaction terms modulated by contralateral M1 activity or ipsilateral M1 activity were used to model the direct and non-direct corticospinal networks. Model 1 is the full model that contains the two interaction terms and model 2 only contains the two single terms. Model 3 contains the interaction term modulated by contralateral M1 activity and model 4 contains the other interaction term. See S-9 for model equations. The $\mathrm{R}^{2}$ values were adjusted (see Methods). 
Figure 4. Correlation between the involvement of the direct or non-direct corticospinal networks in hand movement and individual variability in the degree of hand preference. For each individual, we computed the weights for each of the single terms and interaction terms. Whether each component modelling the direct or non-direct corticospinal network contributed to prediction of SHc activity was tested by a simple linear regression analysis (S-9). The black lines indicate significant while the grey lines indicate nonsignificant components (left panels). The scatter plots represented the relationship between the weight for each component ( $\mathrm{x}$ axis) and the EHI score (y axis). a. The model for RHM. b. The model for LHM. 


\section{Supplementary information}

\section{S-1. Echo planar imaging acquired with multiple slices along a sagittal plane.}

This protocol allowed the scanning of regions of interest in one single volume including M1 in bilateral hemispheres and the cervical spinal cord. The left panel shows the sagittal planes along the longitudinal body axis (i.e. rostro-caudal) covering the brain hemisphere and the spinal cord. $\mathrm{A}=$ anterior side. $\mathrm{P}=$ posterior side. Right panels show axial images of bilateral M1s (see the while line at the level of hand area in M1, left panel) and the spinal cord (see while line at the segmental level of $\mathrm{C} 7$, left panel) that were reconstructed from the raw data consisting of the sagittal slices. $\mathrm{L}=$ left side. $\mathrm{R}=$ right side. Upper and lower side of the axial images correspond to the anterior and posterior side of the brain or the ventral and dorsal side of the spinal cord. $\mathrm{V}=$ ventral side. $\mathrm{D}=$ dorsal side. For display purposes, the axial view of the reconstructed images has been magnified by 3 . 


\section{S-2. Segment-specific and side-specific activity in the spinal cord during hand movement.}

Spinal activation maps at the segments of C7-Th1 from a representative participant (left panels, uncorrected $\mathrm{p}<0.05$ for display purposes) during RHM (upper panel) and LHM (lower panel). L $=$ left side $\mathrm{R}=$ right side. Bar graphs (right panels) represent the mean and standard error of the mean of beta estimate values (arbitrary unit, a.u.) averaged across participants in contralateral or ipsilateral SHc at the segmental level of $\mathrm{C} 5$ or $\mathrm{C} 7-\mathrm{Th} 1$ with respect to the moving hand. * p $<$ 0.05. n.s. $=$ not significant. contra-SHc $=$ contralateral SHc. ipsi-SHc $=$ ipsilateral SHc.

The effects of SEGMENT (C5/C7-Th1), SIDE (contralateral/ipsilateral) and HAND (right hand/left hand) on SHc activity were computed using three way ANOVA. The main effect of SEGMENT (i.e., C7Th1 activity was greater than $\mathrm{C} 5$ activity; $\left.\mathrm{F}_{(1,12)}=6.72 ; \mathrm{p}=0.024\right)$ and the interaction effect of SIDE * HAND $\left(\mathrm{F}_{(1,12)}=5.71 ; \mathrm{p}=0.034\right)$ were significant. See also the results for the other interaction terms in the ANOVA table. In a confirmatory volume of interest (VOI) analysis, right and left SHc at the C5 segment did not show increased activity during either RHM or LHM ( $p$ > 0.4 for all VOIs). During RHM, the C7-Th1 segment showed higher activity in right ipsilateral SHc $(p=0.013)$, but not in left contralateral SHc $(p=0.44)$, than did the C5 segment. During RHM, The C7-Th1 segment showed greater activity in ipsilateral SHc than in contralateral SHc $(p=0.008)$. During LHM, the C7-Th1 segment showed higher activity than the C5 segment in both contralateral SHc $(p=0.020)$ and ipsilateral SHc $(p=0.040)$. Comparable activity was observed between ipsilateral SHc and contralateral SHc $(\mathrm{p}=0.72)$. Thus, both RHM and LHM induced the segment-specific contralateral SHc activity. 


\section{S-3. Activity in primary motor cortex (M1) contralateral and ipsilateral to the moving}

hand. Voxel-wise, whole-brain analysis was used to investigate activity in M1 in both hemispheres. RHM and LHM activated contralateral M1 as compared to rest. For left M1 during RHM, peak MNI coordinates $(\mathrm{x}, \mathrm{y}, \mathrm{z})=-34,-20,58, \mathrm{~T}=7.94$, family-wise error $(\mathrm{FWE})$ corrected $\mathrm{p}=0.03$. For right M1 during LHM, peak MNI coordinates $(\mathrm{x}, \mathrm{y}, \mathrm{z})=38,-16,52, \mathrm{~T}=$ 7.85, FWE corrected $\mathrm{p}=0.03$. To estimate ipsilateral M1 activity, analysis was performed in the spherical VOIs with $8 \mathrm{~mm}$ radius centred at the peak coordinate of left M1 or right M1. RHM suppressed activity in right ipsilateral M1 relative to rest $(\mathrm{T}=5.35, \mathrm{FWE}$ corrected $\mathrm{p}=0.04)$, while LHM did not induce suppression of left ipsilateral M1 activity (uncorrected $\mathrm{p}>0.1$ ).

VOI analysis was used to examine different activities between bilateral M1 in the side contralateral or ipsilateral to the moving hand. The beta estimate values were computed within the $8 \mathrm{~mm}$ sphere centred at the peak coordinate of left or right M1. Bar graphs represent the mean and standard error of the mean of beta estimate values (arbitrary unit, a.u.) averaged across participants in contralateral or ipsilateral M1 with respect to the moving hand. ${ }^{*} \mathrm{p}<0.05$. contraM1 = contralateral M1. ipsi-SHc = ipsilateral M1. Activity was higher in contralateral M1 than in ipsilateral M1 during either RHM or LHM (paired t-test of left M1 vs. right M1 during RHM, $\mathrm{p}=0.0002$; paired t-test of left M1 vs. right M1 during LHM, $\mathrm{p}=0.0002)$. Reduction of activity in ipsilateral M1 during RHM was greater than during LHM (paired t-test of left M1 vs. right $\left.\mathrm{M} 1, \mathrm{t}_{12}=-2.54, \mathrm{p}=0.026\right)$ 
S-4. Correlation coefficient in the contralateral M1-SHc network during hand movement

relative to rest. Bar graphs represent the mean and standard error of the mean of the correlation coefficient $(r)$ between contralateral M1 activity and ipsilateral SHc activity during hand movement or rest $(\mathrm{RHM}, r=0.75 \pm 0.04$; rest, $r=0.09 \pm 0.11$. LHM, $r=0.64 \pm 0.06$; rest, $r=$ $0.014 \pm 0.09)$. Correlation coefficient $(r)$ was higher during hand movement relative to rest, regardless of the moving hand (RHM vs. rest, $p=0.033$. LHM vs. rest, $p=0.043$ ). 


\section{S-5. Correlation coefficient in the ipsilateral M1-SHc network during hand movement}

relative to rest. Bar graphs represent the mean and standard error of the mean of the correlation coefficient $(r)$ between ipsilateral M1 activity and ipsilateral SHc activity during hand movement or rest (RHM, movement, $r=0.065 \pm 0.13$; rest, $r=0.049 \pm 0.11$. LHM, movement, $r=-0.29 \pm$ 0.09 ; rest, $r=0.06 \pm 0.10)$. LHM increased the correlation coefficient $(r)$ in an inverse manner during LHM (movement vs. rest, $\mathrm{p}=0.010$ ), whereas RHM did not affect the coefficient (movement vs. rest, $\mathrm{p}=0.78$ ). 
S-6. Contralateral M1 activity and effective connectivity of the ipsilateral network between M1 and SHc.

Relationship between contralateral M1 activity (x axis) and effective connectivity of the ipsilateral M1-ipsilateral SHc network (y axis), in which each dot indicates a single participant's data. a. The higher the right contralateral M1 activity, the greater the negative value of the effective connectivity of the ipsilateral M1-ipsilateral SHc network during LHM (right panel, correlation coefficient, $r=-0.58, \mathrm{p}=0.03$; simple linear regression analysis, regression slope $=$ $0.58 ; 95 \%$ confidence intervals -0.57 to 0.13$)$. b. Such a relationship was not evident between contralateral M1 activity and effective connectivity of the ipsilateral M1-ipsilateral SHc network during RHM (left panel, $\mathrm{p}=0.39$ ). 


\section{S-7. Functional connectivity and effective connectivity between bilateral M1s.}

Simple correlation analysis was performed between bilateral M1 activity during hand movement or rest. The mean and standard error of the mean of the correlation coefficient $(r)$ are as follows: RHM, movement, $r=-0.07 \pm 0.17$; rest, $r=0.027 \pm 0.11$. LHM, movement, $r=-0.095 \pm 0.12$; rest, $r=0.013 \pm 0.08$. Task induced modulation of interhemispheric functional connectivity was not found during either hand movement (Wilcoxon signed-rank test, RHM, $\mathrm{p}=0.55$; LHM, $\mathrm{p}=$ $0.57)$.

Simple linear regression analysis was computed between bilateral M1 activity during hand movement or rest. The mean and standard error of the mean of the regression slope are as follows: $\mathrm{RHM}$, slope $=-0.13 \pm 0.11$; rest, slope $=0.03 \pm 0.08$. LHM, slope $=-0.04 \pm 0.26$; rest, slope $=0.03 \pm 0.14)$. Neither RHM nor LHM modulated effective connectivity between bilateral M1 (Wilcoxon signed-rank test, RHM, $\mathrm{p}=0.38 ; \mathrm{LHM}, \mathrm{p}=0.50$ ). 


\section{S-8. Models of activity in ipsilateral SHc during LHM.}

We examined whether full models with interaction of activity between bilateral M1s - activity (contralateral M1) * activity (ipsilateral M1)—or simpler models consisting of activity (contralateral M1) or activity (ipsilateral M1) either explained ipsilateral SHc activity during left hand movement. Multiple regression analysis showed that neither type of model was significant $(\mathrm{p}>0.4)$. Information regarding AIC or BIC was not computed in this analysis because it was evident that no models explained SHc activity. The "+" indicates that the corresponding term was included in the models, the "-" indicates that the term was not included. Adjusted R ${ }^{2}, \mathrm{~F}$ values, and $\mathrm{p}$ values are reported. 


\section{S-9. Network model analysis of connectivity between bilateral M1 and ipsilateral SHc during LHM.}

We tested whether the interactions between connectivity of contralateral M1-ipsilateral SHc and connectivity of ipsilateral M1-ipsilateral SHc may mediate modulation of activity between SHc and bilateral M1. The equation for models that contained all terms with interactions is as follows: $\operatorname{activity}(\mathrm{SHc})=\alpha * \operatorname{connectivity}(\operatorname{contralateral~M1-ipsilateral~SHc}) *$ activity $($ contralateral M1 $)+$ $\beta *$ connectivity(ipsilateral M1 - ipsilateral SHc) $*$ activity(ipsilateral M1) $+\gamma_{1} *$ interaction $\{[$ connectivity(contralateral M1-ipsilateral SHc)* connectivity(ipsilateral M1-ipsilateral SHc)] * activity(contralateral M1) $\}+\gamma_{2} *$ interaction $\{[$ connectivity(contralateral M1-ipsilateral SHc) * connectivity(ipsilateral M1-ipsilateral SHc)] * activity(ipsilateral M1) $\}+\varepsilon$, where $\alpha, \beta, \gamma_{1}$ and $\gamma_{2}$ are weights for explanatory variables and $\varepsilon$ indicates residual errors.

We performed the group-level estimate of the likely network models including the interaction terms that may explain SHc activity (see also Fig. 3). The models were constructed so that the same weights were assigned to all participants for each of the interaction terms and the single terms. The table shows a list of all the model equations, labelled "a" through "m", with included terms indicated with "+". Adjusted $\mathrm{R}^{2}, \mathrm{~F}$ values, and $\mathrm{p}$ values are listed for all of the models. AIC and BIC were also computed to estimate the most likely model. The models with and without the interaction terms were compared (paired comparisons are labelled " $\alpha$ " through " $\delta ")$, and the differences in adjusted $\mathrm{R}^{2}\left(\Delta \mathrm{R}^{2}\right)$ are reported. The comparison between models was considered to be statistically significant if $\mathrm{R}^{2}$ was beyond the threshold at the $95 \%$ confidence intervals computed from simulations using bootstrap procedures. All of the comparisons except for $\delta$ (both of the interaction terms modulated by activity[contralateral M1] and activity[ipsilateral M1] vs. the interaction modulated only by activity[contralateral M1]) reached 
significance. The interaction term modulated by activity(contralateral M1) was the most effective in explaining SHc activity, and comparable to the two interaction terms. The model ranking results using AIC and BIC support the model composed of only this interaction term as the most likely model. 


\section{S-10. Simulation analysis of SHc activity during hand movement.}

The simulation analysis was performed to estimate the individual participants' differences in the involvement of the direct and non-direct corticospinal networks. Our previous analyses (see S-6) suggested that the non-direct corticospinal network model modulated by contralateral M1 activity was the most likely one for LHM. It is also possible that the contralateral or ipsilateral direct corticospinal network models might participate in controlling LHM (see Fig. 2). Hence, those three components were all included in the model here. We used the same model for both right hand movement (RHM) and LHM. The equation is as follows: activity $(\mathrm{SHc})=\alpha$ * connectivity (contralateral M1-ipsilateral SHc) * activity (contralateral M1) $+\beta *$ connectivity(ipsilateral M1-ipsilateral SHc) * activity(ipsilateral M1) $+\gamma_{1} *$ interaction $\{[$ connectivity(contralateral M1- ipsilateral SHc) * connectivity(ipsilateral -M1-ipsilateral $\mathrm{SHc})] *$ activity $($ contralateral M1) $\}+\varepsilon$. The weights for each term were determined so that the residual errors were minimized for each individual in the model. Computed information regarding adjusted $\mathrm{R}^{2}, \mathrm{~F}$ values, $\mathrm{p}$ values, and AIC or BIC is listed for all models.

First, we tested if the components could predict ipsilateral SHc activity with use of the individually optimized weights for RHM or LHM. The component for the contralateral direct corticospinal network $\left(\mathrm{R}^{2}=0.34, \mathrm{p}=0.03\right)$ contributed to prediction of ipsilateral SHc activity in RHM more than that for the ipsilateral direct corticospinal network $\left(\mathrm{R}^{2}=0.01, \mathrm{p}=0.65\right)$ or the non-direct corticospinal network $\left(\mathrm{R}^{2}=0.03, \mathrm{p}=0.55\right)$. In the model for LHM, the component for the contralateral direct corticospinal network $\left(\mathrm{R}^{2}=0.66, \mathrm{p}=0.00075\right)$ or the non-direct corticospinal network $\left(\mathrm{R}^{2}=0.70, \mathrm{p}=0.00034\right)$ contributed to prediction of SHc activity, but the component for the ipsilateral direct corticospinal network did not $\left(\mathrm{R}^{2}=0.03, \mathrm{p}=0.58\right)$. There was no evidence that prediction accuracy was higher in the combination of the two components 
for the contralateral direct corticospinal network plus the non-direct corticospinal network $\left(\mathrm{R}^{2}=\right.$ $0.817, \mathrm{p}=0.00020)$ than the single component for only the non-direct corticospinal network or the contralateral direct corticospinal network. AIC and BIC estimated that the combination model was the most likely one. 


\section{S-11. Simulation analysis of recruitment of the contralateral direct corticospinal network during hand movement.}

Our previous analyses (see Fig. 3 and S-6) supported the involvement of the contralateral direct corticospinal network during movement of either hand. We examined if the component of the contralateral direct corticospinal network may explain SHc activity to a greater extent during RHM than during LHM. The weight computed in S-6 indicated the degree to which each network component may predict SHc activity. The weights for the contralateral direct corticospinal network were compared between RHM and LHM by Wilcoxon signed-rank test, and were found to be higher during RHM than during LHM $(p=0.0005)$. 


\section{S-12. Functional connectivity and effective connectivity in either hemisphere of primary motor cortex (M1) or between bilateral M1.}

We did not find evidence supporting interhemispheric functional connections between M1s during RHM or LHM in the employed task (S-7). We further sought evidence that participant variability in interhemispheric influences between bilateral M1 might not be associated with the differences in the degree of hand preference between individuals. First, we examined the relationship between preference for the right hand and functions of the interhemispheric M1 projection. Next, we tested the relationship between nonpreference for the left hand and functions of the interhemispheric M1 projection. Both the regression slope and the correlation coefficient $(r)$, estimated through the individual-level analysis, were used as measures of effective connectivity and functional connectivity, respectively. There was no evidence that the EHI score was correlated with interhemispheric effective connectivity or with functional connectivity between bilateral M1 during RHM or LHM ( $p>0.3$ for all analyses). Finally, we investigated whether bilateral M1 activity or interaction between M1 in both hemispheres might be correlated with the degree of hand preference, but these parameters were not correlated with hand preference $(\mathrm{p}>0.2$ for all analyses $)$. 


\section{Figures}

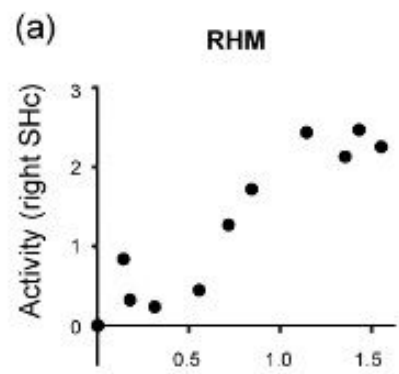

Activity (left M1)

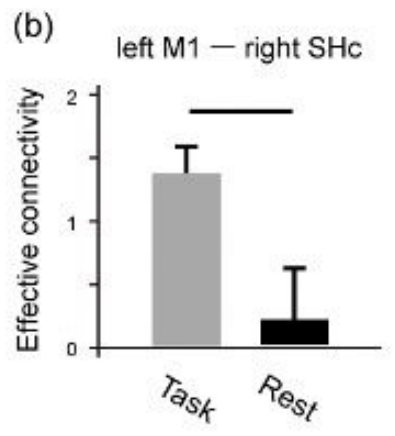

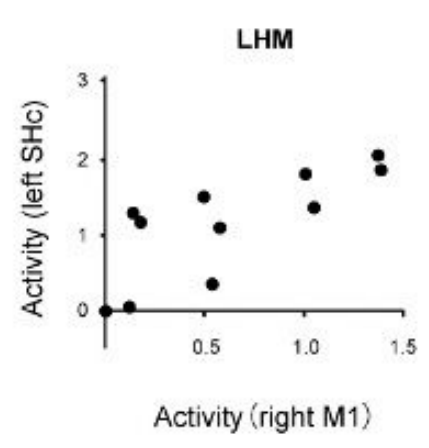

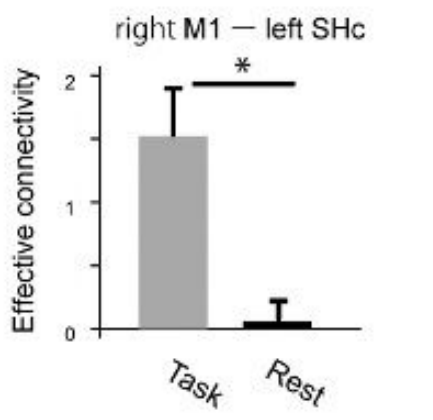

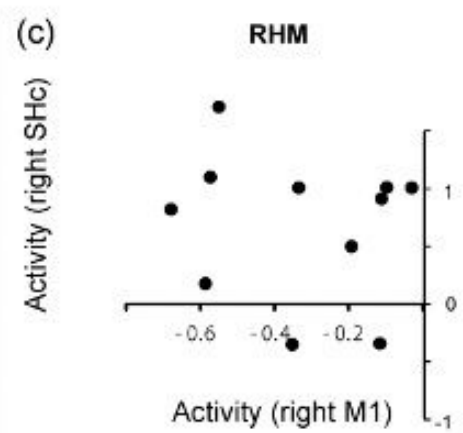

(d) right $\mathrm{M} 1-$ right $\mathrm{SHc}$

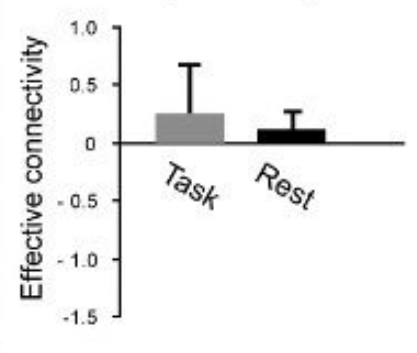

LHM
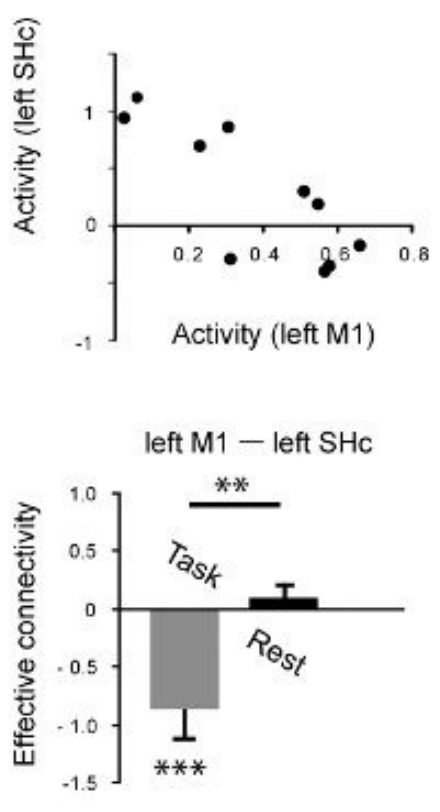

Figure 1

Asymmetric recruitment of the ipsilateral corticospinal network during hand movement. a. Correlation between activity in contralateral M1 (x axis) and ipsilateral SHc (y axis) during RHM (left panel) or LHM (right panel) in a representative participant. Dots indicate the mean activity at each time point during hand movements. b. Effective connectivity (contralateral M1-ipsilateral SHc) for RHM and LHM during the task and during rest periods. ${ }^{*} p<0.001$. c. Correlation between ipsilateral M1 activity ( $x$ axis) and ipsilateral SHc activity (y axis) in the same representative participant. d. Effective connectivity (ipsilateral M1-ipsilateral SHc) for RHM and LHM during the task and rest periods ( $* \star p=0.03$, $* \star \star$ indicates $p=$ $0.01)$. 
(a)

$$
\mathrm{R}
$$

M 1

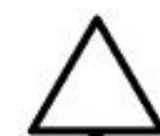

Spinal cord

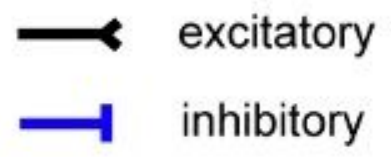

(b)

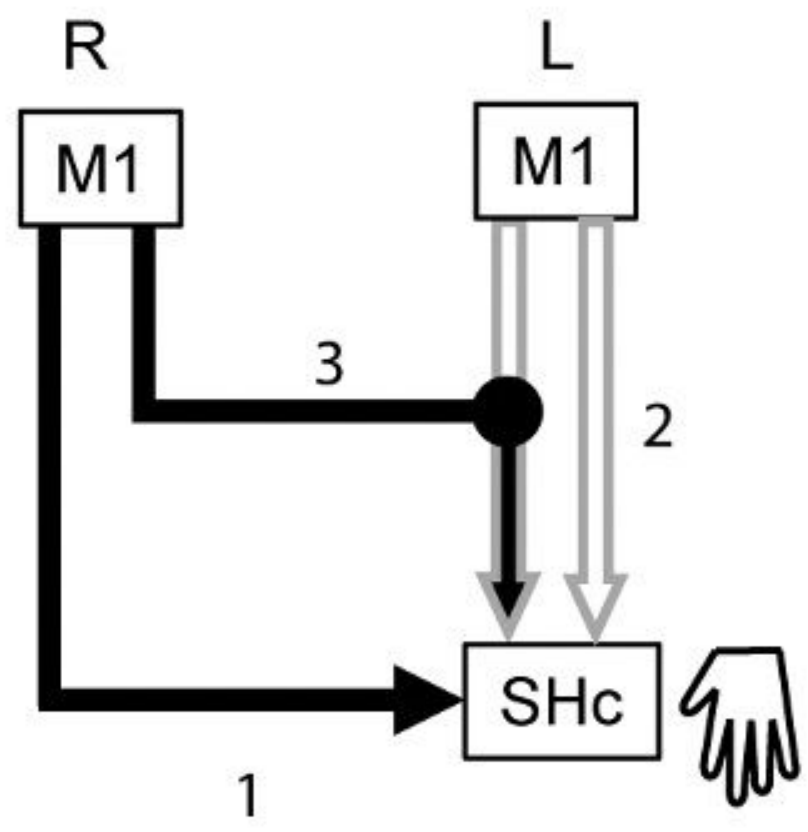

non-direct corticospinal network

interaction of the two projections

Figure 2

Effective connectivity between M1 and SHc and a proposed network model for LHM. a. The positive effective connectivity underpins the net excitatory influences onto the motoneurons (black line; see also Fig. 1b), and negative effective connectivity represents the net inhibitory effects on motoneurons (blue vertical line; see also Fig. 1d). Signals from right contralateral M1 may exert modulation of the negative effective connectivity (black horizontal line, see also S-6) 22. Neurophysiological evidence suggested interaction between effective connectivity of the contralateral M1-SHc and the ipsilateral M1-SHc networks that occurred at the brainstem or the spinal cord 20, 52. b. The direct corticospinal network models were constructed with the single influences from right M1 (black line) or left M1 (white line with gray outline) onto SHc. The ipsilateral direct corticospinal network was operationally considered an alternative model for the contralateral direct network, although evidence did not support involvement of the ipsilateral direct network during hand movement 53. The non-direct corticospinal network model was designed to integrate the influences from left and right M1 onto SHc (black line with gray outline). 


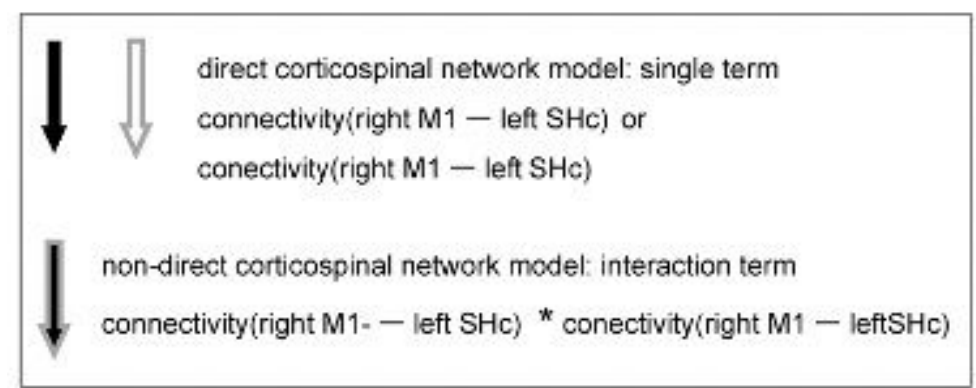

model 1

$\underline{\operatorname{model} 2}$

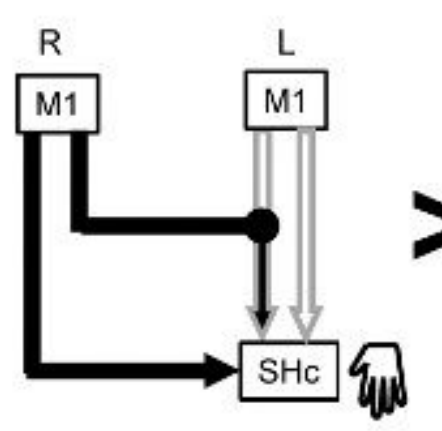

$\mathrm{R}^{2}=0.46$

$(F=3.99, p=0.06$ )

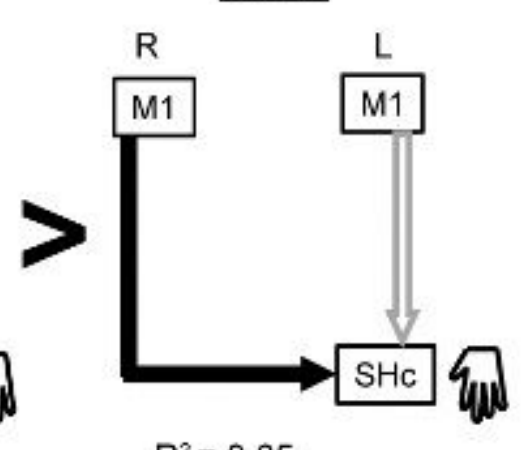

$\mathrm{R}^{2}=0.05$

( $F=1.32, p=0.31$ )

$\underline{\operatorname{model} 3}$

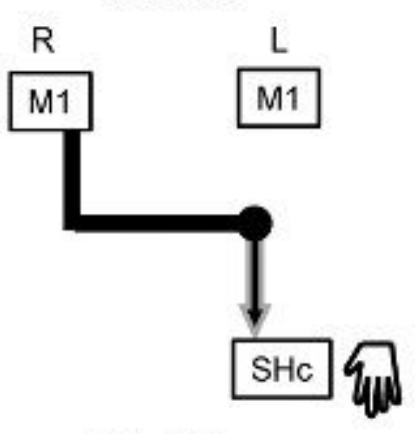

model 4

$\mathrm{R}^{2}=0.48$

( $F=11.9, p=0.005$ )

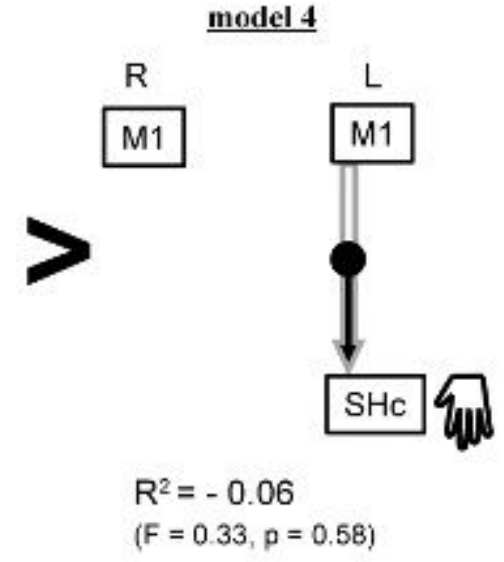

\section{Figure 3}

Network models of SHc activity during LHM with single or interaction terms. Single terms (black line and white line with gray outline) and interaction terms (black line with gray outline) in the connectivity model. According to structural equation modelling, single and interaction terms modulated by contralateral M1 activity or ipsilateral M1 activity were used to model the direct and non-direct corticospinal networks. Model 1 is the full model that contains the two interaction terms and model 2 only contains the two single terms. Model 3 contains the interaction term modulated by contralateral M1 activity and model 4 contains the other interaction term. See S-9 for model equations. The R2 values were adjusted (see Methods). 

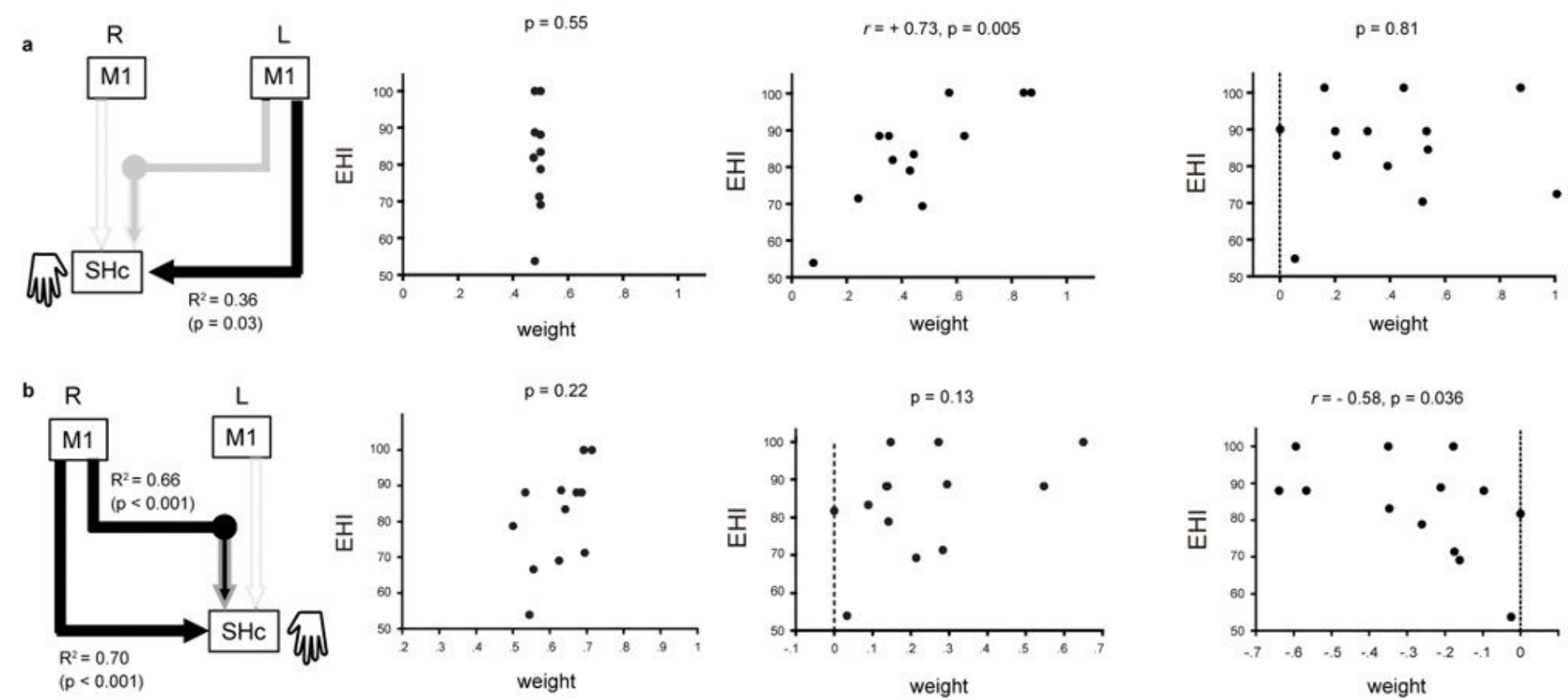

Figure 4

Correlation between the involvement of the direct or non-direct corticospinal networks in hand movement and individual variability in the degree of hand preference. For each individual, we computed the weights for each of the single terms and interaction terms. Whether each component modelling the direct or nondirect corticospinal network contributed to prediction of SHc activity was tested by a simple linear regression analysis (S-9). The black lines indicate significant while the grey lines indicate nonsignificant components (left panels). The scatter plots represented the relationship between the weight for each component ( $x$ axis) and the EHI score (y axis). a. The model for RHM. b. The model for LHM. 